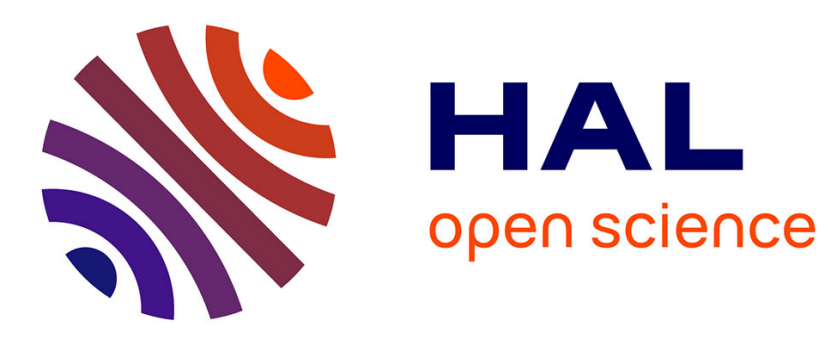

\title{
Sur le dual unitaire de GLr(D)
}

Alexandru Ioan Badulescu, Guy Henniart, Bertrand Lemaire, Vincent

Sécherre

\section{To cite this version:}

Alexandru Ioan Badulescu, Guy Henniart, Bertrand Lemaire, Vincent Sécherre. Sur le dual unitaire de GLr(D). American Journal of Mathematics, 2010, 132 (5), pp.1365 - 1396. 10.1353/ajm.2010.0009 . hal-01822259

\section{HAL Id: hal-01822259 \\ https://hal.science/hal-01822259}

Submitted on 24 Jun 2018

HAL is a multi-disciplinary open access archive for the deposit and dissemination of scientific research documents, whether they are published or not. The documents may come from teaching and research institutions in France or abroad, or from public or private research centers.
L'archive ouverte pluridisciplinaire HAL, est destinée au dépôt et à la diffusion de documents scientifiques de niveau recherche, publiés ou non, émanant des établissements d'enseignement et de recherche français ou étrangers, des laboratoires publics ou privés. 


\title{
SUR LE DUAL UNITAIRE DE $\mathrm{GL}_{r}(D)$
}

\author{
par \\ I. Badulescu, G. Henniart, B. Lemaire \& V. Sécherre
}

\begin{abstract}
Résumé. - Soit $F$ un corps commutatif localement compact non archimédien, et soit $G$ une forme intérieure de $\mathrm{GL}_{n}(F)$. Nous prouvons la conjecture $\mathrm{U} 1$ de Tadić dans le cas où $F$ est de caractéristique non nulle. Ce résultat implique que la description du dual unitaire de $G$ et le transfert de Jacquet-Langlands des représentations unitaires de $G$, tous deux connus pour $F$ de caractéristique nulle, sont vrais en caractéristique quelconque.
\end{abstract}

Abstract. - Let $F$ be a non-Archimedean locally compact field, and let $G$ be an inner form of $\mathrm{GL}_{n}(F)$. We prove Tadić's conjecture U1 when the characteristic of $F$ is positive. This result implies that the description of the unitary dual of $G$ and the Jacquet-Langlands transfer of unitary representations of $G$, both known for $F$ of characteristic zero, are true in any characteristic.

\section{Introduction}

Soient $F$ un corps commutatif localement compact non archimédien et $D$ une $F$-algèbre à division de dimension finie et de centre $F$. On fixe un entier $r \geqslant 1$ et on désigne par $G$ le groupe $\mathrm{GL}_{r}(D)$. Lorsque $F$ est de caractéristique nulle, Tadić a donné dans [31] une description conjecturale du dual unitaire de $G$ - c'est-à-dire de l'ensemble des classes d'isomorphisme de représentations lisses complexes de $G$ qui sont irréductibles et unitaires (ou unitarisables) - et a montré grâce à [17] que la validité de cette description repose sur celle de deux assertions, notées U0 et U1 (voir plus bas). Les travaux de Badulescu $[3,4]$ montrent que, lorsque $F$ est de caractéristique non nulle, la description proposée par Tadić a encore un sens et que la réduction de cette description à U0 et U1 est encore

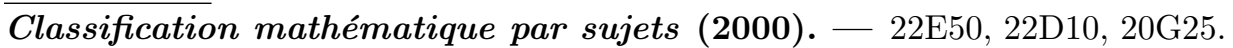

Mots clefs. - Algèbre centrale simple, dual unitaire, conjecture U1, correspondance de JacquetLanglands, corps locaux proches. 
valable. Dans le cas où $D$ est égale à $F$ et de caractéristique quelconque, les assertions U0 et U1 ont été démontrées il y a plus de vingt ans par Bernstein [11] et Tadić [30]. Dans le cas où $F$ est de caractéristique nulle et $D$ quelconque, elles ont été récemment démontrées par Sécherre [28] et Badulescu et Renard [7]. Dans ces deux cas, la classification du dual unitaire de $G$ est donc établie. Il reste à traiter le cas où $F$ est de caractéristique non nulle et $D$ quelconque, ce qui est l'objet du présent article.

On se débarrasse rapidement de l'assertion U0, qui s'énonce comme suit :

(U0) L'induite parabolique normalisée d'une représentation irréductible unitaire d'un sous-groupe de Levi de $G$ est irréductible.

On verra en effet (au paragraphe 2.4) que la preuve qui en est donnée dans [28] lorsque $F$ est de caractéristique nulle est valable en caractéristique quelconque. Il ne reste donc qu'à prouver $\mathrm{U} 1$ dans le cas où $F$ est de caractéristique non nulle, ce qui constitue le résultat principal de cet article : voir le théorème 3.18.

Afin d'énoncer U1 et d'exposer le principe des deux démonstrations que nous en proposons, il est nécessaire d'introduire quelques notations. Soient $k, n \geqslant 1$ des entiers dont le produit est égal à $r$ et soit $\sigma$ une représentation irréductible de carré intégrable (modulo le centre) du groupe $H=\mathrm{GL}_{n}(D)$. On désigne par $M$ et $P$ les sous-groupes de $G$ constitués respectivement des matrices diagonales et triangulaires supérieures par blocs de taille $n$, et on identifie $M$ au produit de $k$ copies de $H$. Pour tout entier $i \in\{1, \ldots, k\}$, on pose :

$$
a_{i}=\frac{k+1}{2}-i
$$

et on note $\nu$ le caractère complexe de $H$ égal à la valeur absolue normalisée de la norme réduite. Au moyen de la correspondance de Jacquet-Langlands, on associe à $\sigma$ un entier $s=s(\sigma) \geqslant 1$ (voir les paragraphes 2.1 et 2.2). Le produit tensoriel $\nu^{s a_{1}} \sigma \otimes \cdots \otimes \nu^{s a_{k}} \sigma$ est une représentation de $M$ dont l'induite parabolique normalisée à $G$ suivant $P$ possède un unique quotient irréductible, que l'on note $u(\sigma, k)$. L'assertion U1 s'énonce alors :

(U1) Les représentations $u(\sigma, k)$ sont unitaires.

Dans cet article, nous présentons deux preuves de U1 dans le cas où $F$ est de caractéristique non nulle. La première, exposée au chapitre 3 , consiste à se ramener au cas où $F$ est de caractéristique nulle au moyen de la méthode des corps proches. La seconde, qui nous a été suggérée par Tadić, consiste à ramener la preuve de U1 à celle d'une assertion auxiliaire $\mathrm{U}$ (voir plus bas) puis à ramener la preuve de $\mathrm{U}$ au cas où $D$ est égale à $F$ au moyen de la théorie des types simples ; nous la décrivons brièvement au chapitre 4 . 
Décrivons maintenant notre première preuve de U1, en commençant par rappeler le principe de la méthode des corps proches $([\mathbf{1 6}]$ et $[\mathbf{2 0}])$. On suppose que $F$ est de caractéristique non nulle notée $p$, et on note $d$ la racine carrée de la dimension de $D$ sur $F$. Pour tout entier $m \geqslant 1$, il existe une extension finie $F^{\prime}$ du corps des nombres $p$-adiques et un isomorphisme d'anneaux :

$$
\lambda_{F, F^{\prime}}^{m}: \mathfrak{o}_{F} / \mathfrak{p}_{F}^{m} \rightarrow \mathfrak{o}_{F^{\prime}} / \mathfrak{p}_{F^{\prime}}^{m}
$$

où $\mathfrak{o}$ et $\mathfrak{p}$ désignent respectivement l'anneau des entiers du corps considéré et son idéal maximal. Étant donnée une telle extension $F^{\prime}$ et un tel isomorphisme d'anneaux (1.2), il existe, à isomorphisme près, une unique $F^{\prime}$-algèbre à division $D^{\prime}$ de centre $F^{\prime}$, ayant la même dimension et le même invariant de Hasse que $D$. On choisit des uniformisantes $\varpi_{F}$ et $\varpi_{F^{\prime}}$ de $F$ et $F^{\prime}$ dont les classes se correspondent par l'isomorphisme (1.2), puis on choisit des uniformisantes $\varpi_{D}$ et $\varpi_{D^{\prime}}$ de $D$ et $D^{\prime}$ telles que $\varpi_{D}^{d}=\varpi_{F}$ et $\varpi_{D^{\prime}}^{d}=\varpi_{F^{\prime}}$. Il existe alors un unique isomorphisme d'anneaux :

$$
\lambda_{D, D^{\prime}}^{m}: \mathfrak{o}_{D} / \mathfrak{p}_{D}^{m d} \rightarrow \mathfrak{o}_{D^{\prime}} / \mathfrak{p}_{D^{\prime}}^{m d}
$$

prolongeant $\lambda_{F, F^{\prime}}^{m}$ et faisant se correspondre les classes de $\varpi_{D}$ et de $\varpi_{D^{\prime}}$. Pour tout entier $l \geqslant 1$, on désigne par $K^{l}$ le sous-groupe de $G$ constitué des matrices à coefficients dans $\mathfrak{o}_{D}$ et congrues à l'identité modulo $\mathfrak{p}_{D}^{l d}$, et l'on désigne par $\mathcal{H}^{l}$ l'algèbre de Hecke constituée des fonctions complexes sur $G$ qui sont bi-invariantes par $K^{l}$ et à support compact. On note $G^{\prime}$ le groupe $\mathrm{GL}_{r}\left(D^{\prime}\right)$, et on définit de façon analogue des sous-groupes $K^{\prime l}$ et des algèbres de Hecke $\mathcal{H}^{\prime l}$ pour $l \geqslant 1$. Si $m$ est supérieur ou égal à $l$, l'isomorphisme (1.3) et le choix de $\varpi_{D}$ et $\varpi_{D^{\prime}}$ permettent de définir, par l'intermédiaire des décompositions de Cartan pour $G$ et $G^{\prime}$, un isomorphisme de $\mathbb{C}$-espaces vectoriels :

$$
\zeta_{G, G^{\prime}}^{m, l}: \mathcal{H}^{l} \rightarrow \mathcal{H}^{\prime l}
$$

On sait d'après $[\mathbf{3}]$ que, si $m$ est assez grand (en un sens ne dépendant que des entiers $l, r$ et de $D$; voir le théorème 3.8), alors (1.4) est non seulement un isomorphisme d'espaces vectoriels, mais un isomorphisme d'algèbres. (On sait même d'après [21] que, si $D$ est égale à $F$, le choix $m=l$ convient.) Dans ce cas, si l'on note $\mathcal{R}^{l}$ la catégorie des représentations de $G$ engendrées par leurs vecteurs fixes sous $K^{l}$ et $\mathcal{R}^{\prime l}$ la catégorie obtenue en remplaçant $D$ par $D^{\prime}$, l'isomorphisme (1.4) induit une équivalence de catégories :

$$
\zeta_{G, G^{\prime}}^{m, l}: \mathcal{R}^{l} \rightarrow \mathcal{R}^{l l}
$$


Cette équivalence induit une bijection, que l'on note encore $\boldsymbol{\zeta}_{G, G^{\prime}}^{m, l}$, entre l'ensemble des classes d'isomorphisme de représentations irréductibles de $G$ de niveau inférieur à $l$ (c'està-dire ayant un vecteur non nul fixé par $K^{l}$ ) et l'ensemble des classes d'isomorphisme de représentations irréductibles de $G^{\prime}$ de niveau inférieur à $l$. Si l'on remplace $G$ par $M$, et si l'on note $M^{\prime}$ (resp. $H^{\prime}, P^{\prime}$ ) l'analogue de $M$ (resp. $H, P$ ) obtenu en remplaçant $D$ par $D^{\prime}$, une construction analogue fournit, pourvu que $m$ soit assez grand, un isomorphisme d'algèbres $\zeta_{M, M^{\prime}}^{m, l}$ et une équivalence de catégories $\boldsymbol{\zeta}_{M, M^{\prime}}^{m, l}$. Si $D$ est égale à $F$, on sait d'après [19] que ces équivalences de catégories commutent à l'induction parabolique normalisée, c'est-à-dire qu'on a :

$$
\boldsymbol{\zeta}_{G, G^{\prime}}^{m, l} \circ \boldsymbol{\iota}_{P}^{G}=\boldsymbol{\iota}_{P^{\prime}}^{G^{\prime}} \circ \boldsymbol{\zeta}_{M, M^{\prime}}^{m, l}
$$

où $\boldsymbol{\iota}_{P}^{G}$ et $\boldsymbol{\iota}_{P^{\prime}}^{G^{\prime}}$ désignent respectivement les foncteurs d'induction parabolique normalisée à $G$ suivant $P$ et à $G^{\prime}$ suivant $P^{\prime}$. Pour $D$ quelconque, la démonstration de l'égalité (1.6) donnée dans [19] est encore valable. On procède alors comme suit pour prouver U1. On fixe deux entiers $k, n \geqslant 1$ dont le produit est égal à $r$ et une représentation irréductible de carré intégrable $\sigma$ de $H$, auxquels correspond la représentation irréductible $u(\sigma, k)$ du groupe $G$. On fixe un entier $l \geqslant 1$ supérieur ou égal au niveau de $\sigma$. Il existe alors un entier $m \geqslant l$ tel qu'aux choix de $F^{\prime}, \varpi_{F^{\prime}}$ et $D^{\prime}, \varpi_{D^{\prime}}$ correspondent des isomorphismes d'algèbres $\zeta_{G, G^{\prime}}^{m, l}$ et $\zeta_{M, M^{\prime}}^{m, l}$. Dans ce cas, les équivalences de catégories $\boldsymbol{\zeta}_{G, G^{\prime}}^{m, l}$ et $\boldsymbol{\zeta}_{M, M^{\prime}}^{m, l}$ sont bien définies et la condition (1.6) est vérifiée. On obtient l'égalité :

$$
\boldsymbol{\zeta}_{G, G^{\prime}}^{m, l}(u(\sigma, k))=u\left(\boldsymbol{\zeta}_{H, H^{\prime}}^{m, l}(\sigma), k\right)
$$

entre classes de représentations irréductibles de $G^{\prime}$. La représentation $\boldsymbol{\zeta}_{H, H^{\prime}}^{m, l}(\sigma)$ est de carré intégrable, de sorte que, d'après [7], le membre de droite de (1.7) est une représentation unitaire de $G^{\prime}$. On sait par ailleurs que $u(\sigma, k)$ est une représentation hermitienne, c'està-dire qu'il existe sur son espace une forme hermitienne $h$ non dégénérée et $G$-invariante (qu'on suppose prendre au moins une valeur strictement positive). Par transport de structure, on déduit de l'unitarité de $\boldsymbol{\zeta}_{G, G^{\prime}}^{m, l}(u(\sigma, k))$ que $h$ est définie positive sur l'espace des vecteurs de $u(\sigma, k)$ fixes sous $K^{l}$. Ceci étant valable pour $l \geqslant 1$ quelconque, on en déduit que $u(\sigma, k)$ est unitaire, ce qu'il fallait démontrer.

Décrivons maintenant brièvement notre seconde preuve de U1, dans laquelle le corps $F$ est supposé de caractéristique quelconque. Soit $k \geqslant 2$ un entier tel que $2 k$ divise $r$ et soit $\sigma$ une représentation irréductible de carré intégrable de $\mathrm{GL}_{n}(D)$, avec $r=2 k n$. Dans $[30,32]$, Tadić introduit l'assertion suivante : 
(U) L'induite parabolique normalisée de $u(\sigma, k+1) \otimes u(\sigma, k-1)$ à $G$ est irréductible.

Notons que, dans le cas où $F$ est de caractéristique nulle et $D$ quelconque, cette assertion est une conséquence immédiate de U0 et $\mathrm{U} 1$, et que, dans le cas où $D$ est égale à $F$ et de caractéristique quelconque, elle a été prouvée par Tadić dans [30]. Dans tous les cas, d'après une idée qu'on trouve esquissée dans [32] (voir le paragraphe 4.1), la conjonction de U0 et de U entraîne U1, de sorte que, pour prouver U1, il nous suffit de prouver U. D'après ce qui précède, il nous suffit même de le faire dans le cas où $F$ est de caractéristique non nulle, et pour cela, on peut se ramener au cas où $F$ est de caractéristique nulle au moyen de la méthode des corps proches. Nous avons préféré présenter une méthode différente, qui nous a été suggérée par Tadić, analogue à celle employée dans [28] et consistant à se ramener au cas où $D$ est égale à $F$ au moyen de la théorie des types de Bushnell et Kutzko. Soulignons que cette méthode de réduction, tout comme celle des corps proches exposée plus haut, repose sur des isomorphismes d'algèbres de Hecke.

La classification du dual unitaire de $G$ est donc maintenant établie pour un corps $F$ de caractéristique quelconque.

Tous les résultats présentés dans cet article sont déjà connus dans le cas où $F$ est de caractéristique nulle. Certains d'entre eux, dont la correspondance de Jacquet-Langlands, le sont aussi dans le cas où $F$ est de caractéristique non nulle. Au bout du compte, on montre qu'ils sont tous vrais quelle que soit la caractéristique de $F$. Au chapitre 2, on rappelle la correspondance de Jacquet-Langlands et la définition de l'invariant $s$ associé à une représentation irréductible de carré intégrable, on présente la classification de TadićZelevinsky des classes de représentations irréductibles de $G$ en termes des représentations irréductibles de carré intégrable de ses sous-groupes de Levi, et l'on donne la classification du dual unitaire de $G$. On explique également pourquoi la preuve de U0 donnée dans [28] est valable en caractéristique quelconque. (En fait, la démarche de Tadić repose, en plus de U0 et U1, sur trois assertions notées U2, U3 et U4. Ces assertions supplémentaires sont prouvées dans [31], par une démarche valable en toute caractéristique ; voir la remarque 2.7.) Au chapitre 3, on prouve U1 par la méthode des corps proches. Au chapitre 4, on donne une deuxième preuve de U1 par la méthode de Tadić : on explique pourquoi U0 et U impliquent U1, et l'on décrit brièvement une preuve de U. Enfin, au chapitre 5, on présente des conséquences de la classification du dual unitaire de $G$ : la décomposition des représentations $u(\sigma, k)$ suivant la base standard, et la généralisation de la correspondance de Jacquet-Langlands aux représentations irréductibles unitaires. 


\section{Notations et conventions}

1. Toutes les représentations considérées dans cet article sont supposées lisses et à valeurs dans le groupe des automorphismes d'un espace vectoriel sur le corps $\mathbb{C}$ des nombres complexes. Suivant l'abus de langage habituel, nous ne ferons pas de différence entre représentations unitaires et unitarisables. Lorsqu'aucune confusion ne sera possible, il nous arrivera d'identifier une représentation avec sa classe d'isomorphisme, et d'écrire simplement représentation plutôt que classe d'isomorphisme de représentations.

2. Si $G$ est un groupe topologique localement profini, on désigne par $\mathcal{R}(G)$ la catégorie de ses représentations lisses complexes et par $\mathcal{G}(G)$ le groupe de Grothendieck de ses représentations de longueur finie, c'est-à-dire le $\mathbb{Z}$-module libre engendré par l'ensemble des classes d'isomorphisme de représentations irréductibles de $G$. Si $\sigma$ est une représentation de longueur finie de $G$, on désigne par $[\sigma]$ son image dans $\mathcal{G}(G)$. En particulier, si $\sigma$ est irréductible, $[\sigma]$ désigne sa classe d'isomorphisme.

3. Soit $F$ un corps commutatif localement compact non archimédien, de caractéristique résiduelle notée $p$. Par $F$-algèbre à division on entend $F$-algèbre de dimension finie et de centre $F$, dont l'anneau sous-jacent est un corps. Si $K$ est une extension finie de $F$, ou plus généralement une algèbre à division sur une extension finie de $F$, on note $\mathfrak{o}_{K}$ son anneau d'entiers et $\mathfrak{p}_{K}$ l'idéal maximal de $\mathfrak{o}_{K}$.

4. On fixe une $F$-algèbre à division $D$ et on note $d$ son degré réduit, c'est-à-dire la racine carrée de sa dimension. Pour tout entier $n \geqslant 1$, on pose $G_{n}=\operatorname{GL}_{n}(D)$ et $\mathcal{G}_{n}=\mathcal{G}\left(G_{n}\right)$.

\section{Classification des représentations unitaires}

Dans tout ce chapitre, on fixe un entier $r \geqslant 1$, on pose $G=\mathrm{GL}_{r}(D)$ et on note $Z$ le centre de $G$. Une représentation de $G$ sera dite cuspidale si elle est irréductible et si elle possède un coefficient non nul à support compact modulo $Z$. Elle sera dite de carré intégrable si elle est irréductible et unitaire, et si elle possède un coefficient non nul de carré intégrable sur $G / Z$.

2.1. Correspondance de Jacquet-Langlands et invariant $s$. - Un élément $g \in G$ est dit semisimple régulier si son polynôme caractéristique a des racines distinctes dans une clôture algébrique de $F$. Si $\pi$ est une représentation lisse de longueur finie de $G$, elle est admissible et on note $\Theta_{\pi}$ sa fonction-caractère. C'est une fonction complexe définie 
sur l'ensemble des éléments semisimples réguliers de $G$, localement constante et stable par conjugaison. On adopte les mêmes définitions et notations pour $H=\mathrm{GL}_{r d}(F)$. On dit que deux éléments $g \in H$ et $g^{\prime} \in G$ se correspondent s'ils sont semisimples réguliers et s'ils ont le même polynôme caractéristique ; on écrit alors $g \leftrightarrow g^{\prime}$.

On rappelle la correspondance de Jacquet-Langlands montrée dans [17] lorsque $F$ est de caractéristique nulle et dans [3] lorsque $F$ est de caractéristique non nulle.

Théorème 2.1. - Il existe une unique application JL de l'ensemble des classes d'isomorphisme de représentations de carré intégrable de $H$ dans l'ensemble des classes d'isomorphisme de représentations de carré intégrable de $G$ telle que, pour toute représentation de carré intégrable $\pi$ de $H$, on ait :

$$
\Theta_{\pi}(g)=(-1)^{r d-r} \Theta_{\mathbf{J L}(\pi)}\left(g^{\prime}\right)
$$

pour tout couple $\left(g, g^{\prime}\right) \in H \times G$ d'éléments semisimples réguliers tels que $g \leftrightarrow g^{\prime}$. En outre, l'application $\mathbf{J L}$ est bijective.

D'après la classification de Zelevinsky [35], à toute représentation de carré intégrable $\sigma$ de $H$ correspond un entier $k \geqslant 1$ divisant $r d$ et une représentation irréductible cuspidale $\rho$ de $\mathrm{GL}_{m}(F)$, avec $r d=k m$. Dans la terminologie de Zelevinsky, l'entier $k$ est la longueur du segment attaché à $\sigma$. La classe d'isomorphisme de $\pi$ détermine l'entier $k$ et la classe d'isomorphisme de $\rho$.

Définition 2.2. - Si $\rho$ est une représentation cuspidale unitaire de $G$, on note $s(\rho)$ l'entier $k$ associé à la représentation de carré intégrable $\mathbf{J L}^{-1}(\rho)$.

L'invariant $s$ fait son apparition dans [17, Théorème B.2.b] et est intensivement utilisé par Tadić dans [31].

2.2. Classifications des représentations irréductibles. - On désigne par $\nu$ le caractère complexe de $G$ égal à la valeur absolue normalisée de la norme réduite. Si :

$$
\lambda=\left(r_{1}, \ldots, r_{k}\right)
$$

est une famille d'entiers strictement positifs de somme égale à $r$, on désigne par $M_{\lambda}$ et $P_{\lambda}$ les sous-groupes de $G$ constitués respectivement des matrices diagonales et triangulaires supérieures par blocs de tailles $r_{1}, \ldots, r_{k}$ prises dans cet ordre. Le sous-groupe $M_{\lambda}$ est appelé sous-groupe de Levi standard de $G$ associé à $\lambda$ et sera canoniquement identifié au 
produit $G_{r_{1}} \times \cdots \times G_{r_{k}}$. Le sous-groupe $P_{\lambda}$ est un sous-groupe parabolique de $G$ de composante de Levi $M_{\lambda}$. Pour chaque $i \in\{1, \ldots, k\}$, soit $\pi_{i}$ une représentation de $G_{r_{i}}$. On désigne par :

$$
\pi_{1} \times \cdots \times \pi_{k}
$$

l'induite parabolique normalisée de $\pi_{1} \otimes \cdots \otimes \pi_{k}$ à $G$ suivant $P_{\lambda}$. Si les représentations $\pi_{1}, \ldots, \pi_{k}$ sont irréductibles, l'application :

$$
\left(\left[\pi_{1}\right], \ldots,\left[\pi_{k}\right]\right) \mapsto\left[\pi_{1} \times \cdots \times \pi_{k}\right]
$$

est bien définie et induit par linéarité une application de $\mathcal{G}_{r_{1}} \times \cdots \times \mathcal{G}_{r_{k}}$ dans $\mathcal{G}_{r}$.

La classification des représentations irréductibles de $G$ en fonction des représentations de carré intégrable de ses sous-groupes de Levi standard est une forme de la classification de Langlands améliorée grâce aux résultats de Jacquet, de Bernstein et Zelevinsky, et de Deligne, Kazhdan et Vignéras [17] (voir aussi [4] dans le cas de la caractéristique non nulle). Elle s'énonce comme suit. Soit $\lambda$ comme en (2.1) et, pour chaque $i \in\{1, \ldots, k\}$, soit $\left(b_{i}, \sigma_{i}\right)$ un couple constitué d'un nombre réel $b_{i}$ et d'une représentation de carré intégrable $\sigma_{i}$ de $G_{r_{i}}$, de telle sorte que $b_{1} \geqslant \cdots \geqslant b_{k}$. Une représentation de $G$ de la forme :

$$
\nu^{b_{1}} \sigma_{1} \times \cdots \times \nu^{b_{k}} \sigma_{k}
$$

est appelée une représentation standard de $G$. D'après le théorème du quotient de Langlands, la représentation standard (2.4) a un unique quotient irréductible, que l'on note :

$$
L\left(\nu^{b_{1}} \sigma_{1}, \ldots, \nu^{b_{k}} \sigma_{k}\right) .
$$

Toute représentation irréductible de $G$ est isomorphe à un tel quotient, et sa classe d'isomorphisme détermine à permutation près les couples $\left(b_{i},\left[\sigma_{i}\right]\right)$ pour $i \in\{1, \ldots, k\}$.

On peut formuler cette classification en d'autres termes. Pour tout $n \geqslant 1$, on note $\mathcal{J}_{n}$ l'ensemble des classes d'isomorphisme de représentations irréductibles de $G_{n}$ et $\mathcal{D}_{n}$ le sous-ensemble de $\mathcal{J}_{n}$ constitué des classes d'isomorphisme de représentations essentiellement de carré intégrable, c'est-à-dire de carré intégrable à torsion près par un caractère. On note respectivement $\mathcal{J}$ et $\mathcal{D}$ la réunion disjointe des $\mathcal{J}_{n}$ et des $\mathcal{D}_{n}$ pour $n \geqslant 1$. Par le procédé plus haut, tout multi-ensemble $\delta$ d'éléments de $\mathcal{D}$ détermine un élément de $\mathcal{J}$ que l'on note $\mathcal{T}(\delta)$. L'application :

$$
\delta \mapsto \mathcal{T}(\delta)
$$


définit une bijection entre l'ensemble des multi-ensembles d'éléments de $\mathcal{D}$ et l'ensemble J. Dans le cas où $D$ est égale à $F$, cette classification est due à Zelevinsky [35] (voir aussi Rodier [23]). Elle a ensuite été généralisée par Tadić [31], grâce à [17], au cas où $F$ est de caractéristique nulle et $D$ quelconque. Plus précisément, Tadić procède de façon axiomatique, réduisant la classification aux trois propositions suivantes.

(P1) Soit $\lambda$ comme en (2.1) et, pour chaque $i \in\{1, \ldots, k\}$, soit $\pi_{i}$ une représentation irréductible de $G_{r_{i}}$. Alors :

$$
\left[\pi_{w(1)} \times \cdots \times \pi_{w(k)}\right]=\left[\pi_{1} \times \cdots \times \pi_{k}\right]
$$

pour toute permutation $w$ de $\{1, \ldots, k\}$.

(P2) Soient $k, n \geqslant 1$ des entiers dont le produit est égal à $r$, et soit $\rho$ une représentation cuspidale unitaire de $G_{n}$. Si l'on pose $s=s(\rho)$ et si l'on définit des entiers $a_{i}$ par (1.1), la représentation :

$$
\nu^{s a_{1}} \rho \times \cdots \times \nu^{s a_{k}} \rho
$$

a une unique sous-représentation irréductible. Celle-ci est notée $T(\rho, k)$, et elle est de carré intégrable. Toute représentation de carré intégrable de $G$ s'obtient ainsi ; sa classe d'isomorphisme détermine la paire $([\rho], k)$ et est déterminée par elle.

(P3) Soit $\lambda$ comme en (2.1) et, pour chaque $i \in\{1, \ldots, k\}$, soit $\sigma_{i}$ une représentation de carré intégrable de $G_{r_{i}}$. La représentation standard :

$$
\sigma_{1} \times \cdots \times \sigma_{k}
$$

de $G$ est irréductible.

Dans [31], la réduction de la classification (2.6) aux trois propositions ci-dessus est indépendante de la caractéristique de $F$. La proposition P1 est vraie en toute caractéristique (voir $[\mathbf{1 2}, 5.4]$ ). Dans le cas où $D$ est égale à $F$, la proposition $\mathrm{P} 2$ est due à Bernstein et Zelevinsky [35, 9.3], l'invariant $s$ valant toujours 1 . Dans le cas où $D$ est quelconque, elle résulte du cas précédent grâce à la correspondance de Jacquet-Langlands $[\mathbf{1 7}, \mathbf{3}]$. Quant à la proposition P3, elle est démontrée dans $[\mathbf{1 7}]$ dans le cas où $F$ est de caractéristique nulle, et dans [4] dans le cas où $F$ est de caractéristique non nulle. Par conséquent, la classification donnée plus haut est valable pour $F$ de caractéristique quelconque et $D$ quelconque.

Remarque 2.3. - On renvoie également à Mínguez [22]. En particulier, dans le cas où $D$ est égale à $F$, il étend la classification (2.6) aux représentations lisses de $G$ à valeurs 
dans le groupe des automorphismes d'un espace vectoriel sur un corps algébriquement clos $R$ de caractéristique banale - c'est-à-dire ne divisant le pro-ordre d'aucun sous-groupe ouvert compact de $G$. Mentionnons un travail en cours de Mínguez et Sécherre dans lequel il est question d'étendre cette classification aux représentations de $G$ à coefficients dans $R$, sans restriction ni sur $D$, ni sur la caractéristique de $R$. Pour le cas où $D$ est égale à $F$, voir Vignéras [34].

La proposition P2 permet d'étendre la définition de l'invariant $s$ aux représentations de carré intégrable de $G$.

Définition 2.4. - Soit $\sigma$ une représentation de carré intégrable de $G$, et soient $k, n \geqslant 1$ des entiers de produit égal à $r$ et $\rho$ une représentation cuspidale unitaire $\rho$ de $G_{n}$ telle que $\sigma$ soit isomorphe à $T(\rho, k)$. Alors on pose $s(\sigma)=s(\rho)$.

2.3. Classification des représentations irréductibles unitaires. - Soient $k, n \geqslant 1$ des entiers et soit $\sigma$ une représentation de carré intégrable de $G_{n}$. On définit des entiers $a_{i}$ par (1.1) et on pose $s=s(\sigma)$. On pose :

$$
u(\sigma, k)=L\left(\nu^{s a_{1}} \sigma, \ldots, \nu^{s a_{k}} \sigma\right),
$$

qui est une représentation irréductible de $G_{k n}$ et, pour tout nombre réel $\alpha$ strictement compris entre 0 et $1 / 2$, on pose :

$$
\pi(u(\sigma, k), \alpha)=\nu^{s \alpha} u(\sigma, k) \times \nu^{-s \alpha} u(\sigma, k),
$$

qui est une représentation de $G_{2 k n}$.

Remarque 2.5. - Les représentations définies par (2.8) sont irréductibles : c'est une conséquence de la proposition 2.2 et du lemme 2.5 de [31], valables pour $D$ quelconque et $F$ de caractéristique quelconque (voir aussi $[3,4]$ ).

Soit $\mathcal{U}$ l'ensemble des classes d'isomorphisme de toutes les représentations de la forme $u(\sigma, k)$ et $\pi(u(\sigma, k), \alpha)$, où $k, n \geqslant 1$ sont des entiers, $\sigma$ une représentation de carré intégrable de $G_{n}$ et $\alpha$ un nombre réel strictement compris entre 0 et $1 / 2$. Le théorème suivant établit la classification du dual unitaire de $G$.

Théorème 2.6. - Tout produit d'éléments de U est irréductible et unitaire. Inversement, toute représentation irréductible unitaire de $G$ est un produit d'éléments de $\mathcal{U}$, et détermine le multi-ensemble des facteurs de ce produit. 
Dans le cas où $F$ est de caractéristique nulle, ce théorème est démontré par Tadić dans [31], grâce aux résultats récents de Badulescu-Renard [7] et Sécherre [28] (voir plus bas). Dans cet article, nous le prouvons dans le cas où $F$ est de caractéristique non nulle. Considérons les deux conjectures suivantes :

(U0) Soit $\lambda$ comme en (2.1) et, pour chaque $i \in\{1, \ldots, k\}$, soit $\pi_{i}$ une représentation irréductible unitaire de $G_{r_{i}}$. Alors la représentation $\pi_{1} \times \cdots \times \pi_{k}$ est irréductible.

(U1) Les représentations $u(\sigma, k)$ sont unitaires.

Dans le cas où $F$ est de caractéristique nulle, Tadić $[\mathbf{3 1}]$ a réduit la preuve du théorème 2.6 à celle des seules conjectures U0 et U1 grâce aux travaux de [17]. Les travaux de Badulescu $[3,4]$ montrent que cette réduction est encore valable lorsque $F$ est de caractéristique non nulle. Dans le cas où $D$ est égale à $F$ et de caractéristique quelconque, les conjectures U0 et U1 ont été démontrées repectivement par Bernstein [11] et Tadić [30]. Dans le cas où $F$ est de caractéristique nulle et $D$ quelconque, elles l'ont été respectivement par Sécherre [28] et Badulescu et Renard [7]. Il reste donc à prouver les conjectures U0 et U1 dans le cas où $F$ est de caractéristique non nulle et $D$ quelconque.

Remarque 2.7. - Dans [31], Tadić utilise, en plus de U0 et U1, trois assertions notées $\mathrm{U} 2$, U3 et U4 pour obtenir le théorème 2.6. Lorsque $F$ est de caractéristique nulle, il montre que ces assertions sont vraies ou conséquences de U0 et U1. En fait, les arguments des paragraphes 1 à 5 de [31] sont indépendants de la caractéristique de $F$, de sorte que l'assertion U4 est prouvée sans condition dans [31, Proposition 2.3] ; l'assertion U3 est vraie également sans condition par [31, Theorem 5.3] et [30, Proposition 3.8]. Enfin, l'assertion U2, qui dit que les représentations (2.8) sont unitaires, est une conséquence de U0 et U1 et du lemme 4.2 plus loin. En effet, U0 et U1 impliquent qu'elles sont irréductibles quand $\alpha$ est nul, et comme on sait qu'elles le sont aussi pour $\alpha$ strictement compris entre 0 et $1 / 2$, le lemme 4.2 implique qu'elles sont unitaires.

2.4. La conjecture U0. — Dans ce paragraphe, nous expliquons pourquoi la preuve de U0 donnée par Sécherre [28] fonctionne en caractéristique quelconque. Cette preuve se décompose en deux parties indépendantes :

(1) d'une part, on ramène la preuve de U0 à celle d'un cas particulier noté S0, dans lequel on impose une condition sur le support cuspidal de la représentation unitaire induisante (voir plus bas); 
(2) d'autre part, on ramène la preuve de S0 pour $D$ quelconque à celle de $\mathrm{S} 0$ pour $D$ égale à $F$, auquel cas le résultat est déjà connu : voir [11].

Afin d'énoncer la conjecture S0, il est commode d'introduire la notion de représentation irréductible simple d'un sous-groupe de Levi standard de $G$. Soit donc $\lambda$ comme en (2.1) et, pour chaque entier $i \in\{1, \ldots, k\}$, soit $\pi_{i}$ une représentation irréductible de $G_{r_{i}}$. La représentation :

$$
\pi_{1} \otimes \cdots \otimes \pi_{k}
$$

du sous-groupe de Levi standard $M_{\lambda}$ est dite simple s'il existe un diviseur $n$ commun à tous les $r_{i}$ et une représentation cuspidale $\rho$ de $G_{n}$ tels que le support cuspidal de (2.9) soit inertiellement équivalent à la représentation cuspidale $\rho \otimes \cdots \otimes \rho$ de $G_{n} \times \cdots \times G_{n}$. En d'autres termes, la représentation (2.9) est simple s'il existe $n$ et $\rho$ comme ci-dessus tels que $(2.9)$ soit un sous-quotient de l'induite parabolique normalisée de $(\rho \otimes \cdots \otimes \rho) \chi$ à $M_{\lambda}$, où $\chi$ est un caractère non ramifié de $G_{n} \times \cdots \times G_{n}$. La conjecture S0 s'énonce alors comme suit :

(S0) Soit $\lambda$ comme en (2.1) et, pour chaque $i \in\{1, \ldots, k\}$, soit $\pi_{i}$ une représentation irréductible unitaire de $G_{r_{i}}$. On suppose que $\pi_{1} \otimes \cdots \otimes \pi_{k}$ est simple. Alors la représentation $\pi_{1} \times \cdots \times \pi_{k}$ est irréductible.

Pour prouver S0, on utilise :

(1) le résultat de Barbasch et Moy [8, 9] affirmant qu'une représentation irréductible de $\mathrm{GL}_{n}(F)$ possédant des vecteurs non nuls fixes sous l'action d'un sous-groupe d'Iwahori $I$ est unitaire si et seulement si l'espace de ses vecteurs $I$-invariants est unitaire comme module sur l'algèbre de Hecke-Iwahori de $\mathrm{GL}_{n}(F)$ relative à $I$;

(2) la théorie des paires couvrantes de Bushnell et Kutzko [14] et la théorie des types simples pour le groupe $G$ développée dans $[\mathbf{2 5}, \mathbf{2 6}, \mathbf{2 7}, \mathbf{2 9}]$.

Le résultat de Barbasch et Moy est rédigé en caractéristique nulle mais leur démonstration est valable en caractéristique quelconque. Quant à la théorie des paires couvrantes et à la théorie des types simples pour $G$, elles sont toutes les deux valables en caractéristique quelconque. Aussi la preuve de S0 donnée dans [28] lorsque $F$ est de caractéristique nulle est-elle valable en caractéristique quelconque.

Pour prouver que S0 implique U0, on utilise la propriété P1 (voir le paragraphe 2.2) et le fait que, pour toute représentation irréductible unitaire $\pi$ de $G$, il existe une famille 
$\lambda$ comme en (2.1) et, pour chaque $i \in\{1, \ldots, k\}$, une représentation irréductible unitaire simple $\pi_{i}$ de $G_{r_{i}}$, de telle sorte que :

$$
\pi=\pi_{1} \times \cdots \times \pi_{k}
$$

et que $\pi_{i} \otimes \pi_{j}$ soit simple si et seulement si $i=j$. Celui-ci est basé sur la classification de Tadić-Zelevinsky (2.6) des représentations irréductibles de $G$ en termes de multi-ensembles de représentations essentiellement de carré intégrable, établie au paragraphe 2.2, et sur les deux propriétés suivantes, valables en toute caractéristique [31] :

(1) on a $\mathcal{T}(\delta)^{+}=\mathcal{T}\left(\delta^{+}\right)$pour tout multi-ensemble $\delta$ de représentations essentiellement de carré intégrable, où $\pi^{+}$désigne la conjuguée complexe de la contragrédiente d'une représentation $\pi$ et $\delta^{+}$le multi-ensemble constitué des conjuguées complexes des contragrédientes des éléments de $\delta$;

(2) pour deux multi-ensembles $\delta$ et $\delta^{\prime}$ de représentations essentiellement de carré intégrable, si les représentations $\mathcal{T}(\delta)$ et $\mathcal{T}\left(\delta^{\prime}\right)$ sont simples sans que $\mathcal{T}(\delta) \otimes \mathcal{T}\left(\delta^{\prime}\right)$ ne le soit, alors $\mathcal{T}(\delta) \times \mathcal{T}\left(\delta^{\prime}\right)$ est irréductible et égal à $\mathcal{T}\left(\delta+\delta^{\prime}\right)$.

Le premier point est démontré dans $[31, \S 2]$, et le second est une conséquence immédiate de la proposition 2.2 et du lemme 2.5 de [31] (voir aussi [28, Proposition 1.5]), et ils sont tous les deux valables en caractéristique quelconque.

2.5. La conjecture U1. - La preuve de U1 donnée dans [7] se base sur un résultat de [5] utilisant une forme simple de la formule des traces d'Arthur [1], pour l'instant prouvée seulement en caractéristique nulle. Nous donnons dans cet article deux preuves de U1 dans le cas où $F$ est de caractéristique non nulle. La première, exposée au chapitre 3, consiste à se ramener au cas où $F$ est de caractéristique nulle au moyen de la méthode des corps proches. La seconde reprend une idée de Tadić esquissée dans [32] et permettant de réduire la preuve de U1 à celle de la conjecture suivante :

(U) Pour tous entiers $k, n \geqslant 1$ et toute représentation de carré intégrable $\sigma$ de $G_{n}$, le produit $u(\sigma, k+2) \times u(\sigma, k)$ est irréductible.

Plus précisément, d'après Tadić [32], si U0 et U sont vraies, alors U1 l'est aussi. Comme Tadić nous l'a suggéré, un argument analogue à celui utilisé par Sécherre pour prouver U0, basé sur la théorie des types, permet de prouver U. Ceci est expliqué au chapitre 4, où l'on donne également les arguments de Tadić permettant d'obtenir U1 à partir de U0 et $\mathrm{U}$. 


\section{Une première preuve de $\mathrm{U} 1$ en caractéristique non nulle}

Les notations du chapitre précédent sont en vigueur : l'entier $r \geqslant 1$ et la $F$-algèbre à division $D$ sont fixés, et on note $G$ le groupe $\operatorname{GL}_{r}(D)$ et $Z$ le centre de $G$. On fixe une uniformisante $\varpi_{F}$ de $F$, ainsi qu'une uniformisante $\varpi_{D}$ de $D$ telle que $\varpi_{D}^{d}=\varpi_{F}$.

3.1. Algèbres de Hecke. - On pose $K^{0}=\operatorname{GL}_{r}\left(\mathfrak{o}_{D}\right)$ et, pour tout entier $l \geqslant 1$, on note $K^{l}$ le sous-groupe ouvert distingué de $K^{0}$ constitué des matrices congrues à l'identité modulo $\mathfrak{p}_{D}^{l d}$. Si $K$ est un sous-groupe ouvert compact de $G$, on désige par $\mathcal{H}(G, K)$ l'algèbre de Hecke formée des fonctions complexes sur $G$ qui sont bi-invariantes par $K$ et à support compact. Pour $l \geqslant 0$, on pose simplement $\mathcal{H}^{l}=\mathcal{H}\left(G, K^{l}\right)$.

Définition 3.1. - Le niveau d'une représentation $\pi$ de $G$ est le plus petit entier $l \geqslant 0$ tel qu'il existe un vecteur non nul de l'espace de $\pi$ fixé par $\pi(k)$ pour tout $k \in K^{l}$. On le note $l(\pi)$.

On note $d g$ la mesure de Haar sur $G$ qui donne le volume 1 à $K^{0}$. Si $\pi$ est une représentation de $G$ de niveau inférieur à $l$ sur un espace $V$, on désigne par $V^{l}$ l'espace constitué des vecteurs de $V$ fixes sous $K^{l}$. Il est muni d'une structure de $\mathcal{H}^{l}$-module à gauche par :

$$
(f, v) \mapsto f v=\int_{G} f(g) \pi(g) v d g
$$

pour toute fonction $f \in \mathcal{H}^{l}$ et tout vecteur $v \in V^{l}$. Le foncteur associant à une représentation $(\pi, V)$ l'espace $V^{l}$ de ses vecteurs $K^{l}$-invariants induit une bijection entre l'ensemble des classes d'isomorphisme de représentations irréductibles de $G$ de niveau inférieur à $l$ et l'ensemble des classes d'isomorphisme de $\mathcal{H}^{l}$-modules à gauche simples.

3.2. Une base de l'espace vectoriel $\mathcal{H}^{l}$. - D'après $[\mathbf{2 4}, \S 8.2]$, le groupe $G$ est la réunion disjointe des doubles classes $K^{0} a K^{0}$ où a décrit l'ensemble $\mathcal{A}$ constitué des matrices diagonales de $G$ dont les termes diagonaux sont respectivement $\varpi_{D}^{b_{1}}, \ldots, \varpi_{D}^{b_{r}}$, les $b_{i}$ étant des entiers rangés dans l'ordre croissant : c'est la décomposition de Cartan. Fixons un élément $a \in \mathcal{A}$ et un entier $l \geqslant 1$, et posons :

$$
T^{l}=\mathrm{GL}_{r}\left(\mathfrak{o}_{D} / \mathfrak{p}_{D}^{l d}\right) \times \mathrm{GL}_{r}\left(\mathfrak{o}_{D} / \mathfrak{p}_{D}^{l d}\right) .
$$

Pour $x, y \in K^{0}$, la double classe $K^{l} x a y^{-1} K^{l}$ ne dépend que de l'image de $(x, y)$ dans $T^{l}$, ce qui permet de définir $K^{l} \boldsymbol{x} a \boldsymbol{y}^{-1} K^{l}$ pour tout $(\boldsymbol{x}, \boldsymbol{y}) \in T^{l}$. On désigne par $H_{a}^{l}$ l'ensemble des couples $(\boldsymbol{x}, \boldsymbol{y}) \in T^{l}$ tels qu'il existe $(x, y) \in K^{0} \times K^{0}$ dont l'image dans $T^{l}$ soit $(\boldsymbol{x}, \boldsymbol{y})$ 
et tels qu'on ait $x a=a y$; c'est un sous-groupe de $T^{l}$. On note $\widetilde{T}_{a}^{l}$ le quotient de $T^{l}$ par $H_{a}^{l}$. Pour toute partie $W$ de $G$, on note $\mathbf{1}_{W}$ la fonction caractéristique de $W$.

Proposition 3.2. - (1) Pour tous a $\in \mathcal{A}$ et $(\boldsymbol{x}, \boldsymbol{y}) \in T^{l}$, la double classe $K^{l} \boldsymbol{x} a \boldsymbol{y}^{-1} K^{l}$ ne dépend que de l'image de $(\boldsymbol{x}, \boldsymbol{y})$ dans $\widetilde{T}_{a}^{l}$, de sorte qu'on peut définir $K^{l} \widetilde{\boldsymbol{x}} a \widetilde{\boldsymbol{y}}^{-1} K^{l}$ pour tout $(\widetilde{\boldsymbol{x}}, \widetilde{\boldsymbol{y}}) \in \widetilde{T}_{a}^{l}$.

(2) Pour tout $a \in \mathcal{A}$, on a la décomposition:

$$
K^{0} a K^{0}=\coprod_{(\widetilde{\boldsymbol{x}}, \widetilde{\boldsymbol{y}}) \in \widetilde{T}_{a}^{l}} K^{l} \widetilde{\boldsymbol{x}} a \widetilde{\boldsymbol{y}}^{-1} K^{l} .
$$

(3) L'ensemble des $\mathbf{1}_{K^{l} \widetilde{\boldsymbol{x}} a \widetilde{\boldsymbol{y}}^{-1} K^{l}}$, pour $a \in \mathcal{A}$ et $(\widetilde{\boldsymbol{x}}, \widetilde{\boldsymbol{y}}) \in \widetilde{T}_{a}^{l}$, est une base de $\mathcal{H}^{l}$ comme $\mathbb{C}$-espace vectoriel.

Démonstration. - Les affirmations (1) et (2) sont prouvées dans [3, lemme 2.6], et (3) est une conséquence immédiate de (2).

3.3. Représentations hermitiennes et unitaires. - Soit $\pi$ une représentation de $G$, et soit $h$ une forme hermitienne sur l'espace $V$ de $\pi$, c'est-à-dire une forme sesquilinéaire telle que $h\left(v, v^{\prime}\right)=\overline{h\left(v^{\prime}, v\right)}$ pour tous vecteurs $v, v^{\prime} \in V$. (Ici $\bar{z}$ désigne le conjugué d'un nombre complexe $z$.) On dit que $h$ est stable par $\pi$ si :

$$
h\left(v, v^{\prime}\right)=h\left(\pi(g) v, \pi(g) v^{\prime}\right)
$$

pour tout $g \in G$ et tous $v, v^{\prime} \in V$. Le noyau de $h$ est alors un sous-espace de $V$ stable par $G$, de sorte que, si $\pi$ est irréductible et $h$ non nulle, la forme $h$ est non dégénérée.

On dit que $\pi$ est hermitienne s'il existe une forme hermitienne $h$ sur $V$, non dégénérée et stable par $\pi$. On dit qu'elle est unitaire si elle est hermitienne et si l'on peut choisir $h$ définie positive. Dans ce dernier cas, on dit que $h$ est un produit scalaire hermitien sur $V$ stable par $\pi$.

On suppose que $\pi$ est hermitienne, et l'on fixe une forme hermitienne $h$ sur $V$ non dégénérée et stable par $\pi$. Pour tout entier $l \geqslant 0$, la forme $h$ induit par restriction sur le sous-espace $V^{l}$ de $V$ une forme hermitienne que l'on note $h^{l}$. Pour $f \in \mathcal{H}^{l}$, on note $f^{*}$ la fonction $g \mapsto \overline{f\left(g^{-1}\right)}$, qui appartient à $\mathcal{H}^{l}$.

Lemme 3.3. - Pour toute $f \in \mathcal{H}^{l}$, tout $v \in V^{l}$ et tout $v^{\prime} \in V$, on a :

$$
h\left(f v, v^{\prime}\right)=h^{l}\left(v, f^{*} v^{\prime}\right) .
$$


Démonstration. - Il suffit de vérifier cette relation dans le cas où $f$ est la fonction caractéristique de $K^{l} g K^{l}$ pour $g \in G$, auquel cas $f^{*}$ est la fonction caractéristique de $K^{l} g^{-1} K^{l}$. Écrivons $K^{l} g K^{l}$ comme la réunion disjointe des $g_{i} K^{l}$, avec $g_{i} \in G$ pour $1 \leqslant i \leqslant k$. On a :

$$
h\left(f v, v^{\prime}\right)=c \sum_{i=1}^{k} h\left(\pi\left(g_{i}\right) v, v^{\prime}\right)=c \sum_{i=1}^{k} h\left(v, \pi\left(g_{i}^{-1}\right) v^{\prime}\right),
$$

où $c$ désigne le volume de $K^{l}$ relativement à la mesure $d g$. Comme $K^{l} g^{-1} K^{l}$ est la réunion disjointe des $K^{l} g_{i}^{-1}$, on a aussi :

$$
h^{l}\left(v, f^{*} v^{\prime}\right)=\sum_{i=1}^{k} h\left(v, \int_{K^{l}} \pi(k) \pi\left(g_{i}^{-1}\right) v^{\prime} d k\right)=\sum_{i=1}^{k} \int_{K^{l}} h\left(v, \pi(k) \pi\left(g_{i}^{-1}\right) v^{\prime}\right) d k,
$$

ce dont on déduit :

$$
h^{l}\left(v, f^{*} v^{\prime}\right)=\sum_{i=1}^{k} \int_{K^{l}} h\left(\pi\left(k^{-1}\right) v, \pi\left(g_{i}^{-1}\right) v^{\prime}\right) d k=c \sum_{i=1}^{k} h\left(v, \pi\left(g_{i}^{-1}\right) v^{\prime}\right),
$$

ce qui prouve le lemme 3.3.

Un $\mathcal{H}^{l}$-module $W$ est dit hermitien s'il existe une forme hermitienne $\varphi$ sur $W$ non dégénérée et stable par $\mathcal{H}^{l}$, c'est-à-dire vérifiant la relation :

$$
\varphi\left(f w, w^{\prime}\right)=\varphi\left(w, f^{*} w^{\prime}\right)
$$

pour tout $f \in \mathcal{H}^{l}$ et tous $w, w^{\prime} \in W$. Si $\pi$ est hermitienne et de niveau inférieur à $l$, alors le lemme 3.3 appliquée à $f=\mathbf{1}_{K^{l}}$ montre que $h^{l}$ est non dégénérée, donc que le $\mathcal{H}^{l}$-module $V^{l}$ est hermitien.

Remarque 3.4. - Réciproquement, d'après la formule (2.10) de [8], si le $\mathcal{H}^{l}$-module $V^{l}$ est hermitien et si la représentation $\pi$ est engendrée par $V^{l}$ en tant que représentation de $G$, alors $\pi$ est hermitienne.

Proposition 3.5. - Soit $\pi$ une représentation irréductible de $G$ sur un espace $V$.

(1) Soit un entier $l \geqslant l(\pi)$ et soient $h$ et $h^{\prime}$ deux formes hermitiennes non dégénérées sur $V^{l}$ stables par $\mathcal{H}^{l}$. Alors il existe un nombre réel non nul $\alpha$ tel que $h=\alpha h^{\prime}$.

(2) Soient $h$ et $h^{\prime}$ deux formes hermitiennes non dégénérées (i.e. ici non nulles) sur $V$ stables par $\pi$. Alors il existe un nombre réel non nul $\alpha$ tel que $h=\alpha h^{\prime}$. 
Démonstration. - Soit un vecteur $v \in V^{l}$. Comme $h^{\prime}$ est non dégénérée et que $V^{l}$ est de dimension finie, il existe un unique vecteur $v^{\prime} \in V^{l}$ tel que les formes linéaires $w \mapsto h(v, w)$ et $w \mapsto h^{\prime}\left(v^{\prime}, w\right)$ coïncident sur $V^{l}$. L'application $\theta$ de $V^{l}$ dans $V^{l}$ qui à $v$ associe $v^{\prime}$ est linéaire. Grâce à la relation (3.1) pour $h$ et $h^{\prime}$, on voit que $\theta$ est un morphisme non nul de $\mathcal{H}^{l}$-modules. Le lemme de Schur s'applique : comme $V^{l}$ est un $\mathcal{H}^{l}$-module simple, l'algèbre $\operatorname{End}_{\mathcal{H}^{l}}\left(V^{l}\right)$ est une algèbre à division sur $\mathbb{C}$. Puisque $V^{l}$ est de dimension finie sur $\mathbb{C}$, l'algèbre $\operatorname{End}_{\mathcal{H}^{l}}\left(V^{l}\right)$ l'est aussi ; elle est donc isomorphe à $\mathbb{C}$. Aussi $\theta$ est-elle une homothétie, de sorte que $h$ et $h^{\prime}$ sont proportionnelles. Le rapport est un nombre réel non nul car les formes sont hermitiennes et non dégénérées. Le point (2) est une conséquence de (1) puisque $V$ est réunion des sous-espaces $V^{l}$ pour $l \geqslant l(\pi)$.

3.4. Corps proches. - Comme dans la suite le corps de base va changer, nous ajouterons des indices $F$ aux objets construits auparavant : par exemple, on notera $D_{F}$ la $F$-algèbre à division $D$ et $G_{F}$ le groupe $\mathrm{GL}_{r}\left(D_{F}\right)$. Lorsqu'on voudra faire varier le rang du groupe, nous ajouterons un indice $r$ aux objets introduits précédemment pour $r$ fixé : par exemple, pour $l \geqslant 1$, on notera $K_{F, r}^{l}$ le sous-groupe de $G_{F, r}=\mathrm{GL}_{r}\left(D_{F}\right)$ constitué des matrices à coefficients dans $\mathfrak{o}_{D_{F}}$ et congrues à l'identité modulo l'idéal $\mathfrak{p}_{D_{F}}^{l d}$, et on notera $\mathcal{H}_{F, r}^{l}$ l'algèbre de Hecke $\mathcal{H}\left(G_{F, r}, K_{F, r}^{l}\right)$.

Soit $L$ un corps commutatif localement compact non archimédien. Étant donné un entier $m \geqslant 1$, on dit que $F$ et $L$ sont $m$-proches s'il existe un isomorphisme d'anneaux :

$$
\lambda_{F, L}^{m}: \mathfrak{o}_{F} / \mathfrak{p}_{F}^{m} \rightarrow \mathfrak{o}_{L} / \mathfrak{p}_{L}^{m} .
$$

On suppose que $F$ et $L$ sont $m$-proches et l'on fixe un isomorphisme d'anneaux (3.2).

Remarque 3.6. - Pour tout entier $m^{\prime} \geqslant 1$ inférieur à $m$, les corps $F$ et $L$ sont $m^{\prime}$ proches puisque $\lambda_{F, L}^{m}$ induit par passage aux quotients un isomorphisme d'anneaux :

$$
\lambda_{F, L}^{m, m^{\prime}}: \mathfrak{o}_{F} / \mathfrak{p}_{F}^{m^{\prime}} \rightarrow \mathfrak{o}_{L} / \mathfrak{p}_{L}^{m^{\prime}}
$$

L'invariant de Hasse de $D_{F}$ est un nombre rationnel de la forme $k / d$, où $1 \leqslant k \leqslant d$ est un entier premier à $d$. Soit $D_{L}$ une $L$-algèbre à division de centre $L$, de degré réduit $d$ et d'invariant de Hasse $k / d$. D'après la théorie du corps de classes, il n'existe, à isomorphisme près, qu'une seule telle $L$-algèbre à division. On fixe des uniformisantes $\varpi_{F}$ et $\varpi_{L}$ de $F$ et $L$ dont les classes se correspondent par (3.2), et des uniformisantes $\varpi_{D_{F}}$ et $\varpi_{D_{L}}$ de $D_{F}$ et $D_{L}$ telles que $\varpi_{D_{F}}^{d}=\varpi_{F}$ et $\varpi_{D_{L}}^{d}=\varpi_{L}$. Soit $F^{\prime}$ une extension non ramifiée de $F$ de degré $d$ contenue dans $D_{F}$ et normalisée par $\varpi_{D_{F}}$, et soit $L^{\prime}$ une extension non ramifiée de 
$L$ de degré $d$ contenue dans $D_{L}$ et normalisée par $\varpi_{D_{L}}$. La conjugaison par $\varpi_{D_{F}}$ induit sur $F^{\prime}$ un automorphisme de $F$-algèbres égal à $\varphi_{F^{\prime} / F}^{k}$, où $\varphi_{F^{\prime} / F}$ désigne l'automorphisme de Frobenius de l'extension $F^{\prime} / F$, et on a un résultat analogue pour $L^{\prime} / L$. D'après [16, 3.4.1], les corps $F^{\prime}$ et $L^{\prime}$ sont $m$-proches. Plus précisément, l'isomorphisme $\lambda_{F, L}^{m}$ se prolonge de manière unique en un isomorphisme d'anneaux :

$$
\lambda_{F^{\prime}, L^{\prime}}^{m}: \mathfrak{o}_{F^{\prime}} / \mathfrak{p}_{F^{\prime}}^{m} \rightarrow \mathfrak{o}_{L^{\prime}} / \mathfrak{p}_{L^{\prime}}^{m}
$$

par lequel les automorphismes de Frobenius $\varphi_{F^{\prime} / F}$ et $\varphi_{L^{\prime} / L}$ se correspondent. Soit maintenant un élément $x \in \mathfrak{o}_{D_{F}}$, qu'on écrit comme la somme des $\varpi_{D_{F}}^{i} x_{i}^{\prime}$, avec $x_{i}^{\prime} \in \mathfrak{o}_{F^{\prime}}$ et avec $i \in\{0, \ldots, d-1\}$. Pour chaque $i$, on choisit $y_{i}^{\prime} \in \mathfrak{o}_{L^{\prime}}$ tel que les classes de $x_{i}^{\prime}$ et $y_{i}^{\prime}$ se correspondent par $\lambda_{F^{\prime}, L^{\prime}}^{m}$ et on note $y$ la somme des $\varpi_{D_{F}}^{i} y_{i}^{\prime}$ pour $i \in\{0, \ldots, d-1\}$. Alors la classe de $y$ modulo $\mathfrak{p}_{D_{L}}^{m d}$ ne dépend pas des choix effectués, et ce procédé induit un isomorphisme d'anneaux :

$$
\lambda_{D_{F}, D_{L}}^{m}: \mathfrak{o}_{D_{F}} / \mathfrak{p}_{D_{F}}^{m d} \rightarrow \mathfrak{o}_{D_{L}} / \mathfrak{p}_{D_{L}}^{m d}
$$

Plus précisément, il s'agit de l'unique isomorphisme d'anneaux prolongeant $\lambda_{F, L}^{m}$ par lequel les classes de $\varpi_{D_{F}}$ et $\varpi_{D_{L}}$ se correspondent.

Remarque 3.7. - Pour tout entier $m^{\prime} \geqslant 1$ inférieur à $m$, l'isomorphisme (3.4) induit par passage aux quotients un isomorphisme d'anneaux :

$$
\lambda_{D_{F}, D_{L}}^{m, m^{\prime}}: \mathfrak{o}_{D_{F}} / \mathfrak{p}_{D_{F}}^{m^{\prime} d} \rightarrow \mathfrak{o}_{D_{L}} / \mathfrak{p}_{D_{L}}^{m^{\prime} d}
$$

qui n'est autre que celui construit comme ci-dessus à l'aide de (3.3) et de $\varpi_{D_{F}}$ et $\varpi_{D_{L}}$.

3.5. Corps proches et algèbres de Hecke. — La correspondance $\varpi_{D_{F}} \mapsto \varpi_{D_{L}}$ induit naturellement une bijection $\beta: \mathcal{A}_{F} \rightarrow \mathcal{A}_{L}$ (voir le paragraphe 3.2 pour la définition de $\mathcal{A})$. D'après [3, lemme 2.7], l'isomorphisme d'anneaux $\lambda_{D_{F}, D_{L}}^{m}$ induit un isomorphisme de groupes entre $T_{F}^{m}$ et $T_{L}^{m}$ qui induit à son tour, par restriction pour chaque $a \in \mathcal{A}_{F}$, un isomorphisme de groupes entre $H_{F, a}^{m}$ et $H_{L, \beta(a)}^{m}$ puis une bijection entre $\widetilde{T}_{F, a}^{m}$ et $\widetilde{T}_{L, \beta(a)}^{m}$. Grâce à la proposition 3.2, on obtient ainsi un isomorphisme d'espaces vectoriels :

$$
\zeta_{G_{F}, G_{L}}^{m}: \mathcal{H}_{F}^{m} \rightarrow \mathcal{H}_{L}^{m}
$$

D'après la remarque 3.7, pour tout entier $m^{\prime} \geqslant 1$ inférieur à $m$, l'isomorphisme (3.6) induit par restriction un isomorphisme d'espaces vectoriels :

$$
\zeta_{G_{F}, G_{L}}^{m, m^{\prime}}: \mathcal{H}_{F}^{m^{\prime}} \rightarrow \mathcal{H}_{L}^{m^{\prime}}
$$


qui coïncide avec l'isomorphisme $\zeta_{G_{F}, G_{L}}^{m^{\prime}}$ construit comme ci-dessus à l'aide de $\lambda_{F, L}^{m, m^{\prime}}$ et des uniformisantes $\varpi_{D_{F}}$ et $\varpi_{D_{L}}$.

Le théorème suivant, dérivé d'un théorème de Kazhdan [20] concernant les groupes de Chevalley, est prouvé dans $[\mathbf{2 1}]$ lorsque $D_{F}$ est égale à $F$ (et dans ce cas, si $l \geqslant 1$, on peut prendre $m=l$ ). Lorsque $D_{F}$ est quelconque, c'est le théorème $[\mathbf{3}, 2.13]$.

Théorème 3.8. - Soit un entier $l \geqslant 0$. Il existe un entier non nul $m \geqslant l$ possédant la propriété suivante. Étant donnés :

(1) un corps $p$-adique $L$ qui soit $m$-proche de $F$, une uniformisante $\varpi_{L}$ de $L$ et un isomorphisme d'anneaux (1.2) faisant se correspondre les classes de $\varpi_{F}$ et de $\varpi_{L}$;

(2) une L-algèbre à division $D_{L}$ de centre $L$ ayant la même la même dimension et le même invariant de Hasse que $D_{F}$, et une uniformisante $\varpi_{D_{L}}$ telle que $\varpi_{D_{L}}^{d}=\varpi_{L}$; l'isomorphisme d'espaces vectoriels $\zeta_{G_{F}, G_{L}}^{m, l}$ qui s'en déduit est un isomorphisme d'algèbres.

Remarque 3.9. - Le sous-groupe compact maximal $K^{0}$ de $G$ est le groupe des points $\mathfrak{o}_{F}$-rationnels d'un $\mathfrak{o}_{F}$-schéma en groupes affine lisse connexe $\mathcal{K}$ et, pour chaque entier $l \geqslant 1$, le groupe $K^{l}$ est le noyau de la projection canonique (c'est-à-dire de la réduction modulo $\left.\mathfrak{p}_{F}^{l}\right)$ de $\mathcal{K}\left(\mathfrak{o}_{F}\right)$ sur $\mathcal{K}\left(\mathfrak{o}_{F} / \mathfrak{p}_{F}^{l}\right)$. Notons $\kappa_{F}$ le corps résiduel de $F$ et, pour $l \geqslant 1$, désignons par $K_{l}$ le sous-groupe de $K^{0}$ constitué des matrices congrues à l'identité modulo $\mathfrak{p}_{D}^{l}$, de sorte qu'on a $K^{l}=K_{l d}$. Si $d \neq 1$, le $\kappa_{F}$-groupe algébrique $\overline{\mathcal{K}}=\mathcal{K} \times_{\mathfrak{o}_{F}} \kappa_{F}$ n'est pas réductif, et en notant $\overline{\mathcal{K}}_{\text {réd }}$ son quotient réductif maximal, le groupe $K_{1}$ est le noyau de la projection canonique de $\mathcal{K}\left(\mathfrak{o}_{F}\right)$ sur $\overline{\mathcal{K}}_{\text {réd }}\left(\kappa_{F}\right)$. On pourrait simplifier les énoncés ci-dessus en utilisant la filtration $\left(K_{l}\right)_{l \geqslant 1}$ de $K^{0}$ plutôt que la filtration $\left(K^{l}\right)_{l \geqslant 1}$ : si $D$ et $D^{\prime}$ sont deux algèbres à division (de dimension finie sur leurs centres respectifs) telles qu'il existe un isomorphisme d'anneaux :

$$
\lambda: \mathfrak{o}_{D} / \mathfrak{p}_{D}^{m} \rightarrow \mathfrak{o}_{D^{\prime}} / \mathfrak{p}_{D^{\prime}}^{m}
$$

pour un entier $m \geqslant l$, et si $\varpi_{D}$ et $\varpi_{D^{\prime}}$ sont des uniformisantes de $D$ et $D^{\prime}$ dont les classes se correspondent par (3.8), alors on peut, grâce à la décomposition de Cartan pour $G$ et $G^{\prime}=\mathrm{GL}_{r}\left(D^{\prime}\right)$, construire comme ci-dessus un isomorphisme d'espaces vectoriels :

$$
\zeta: \mathcal{H}\left(G, K_{l}\right) \rightarrow \mathcal{H}\left(G^{\prime}, K_{l}^{\prime}\right),
$$

où $K_{l}^{\prime}$ est défini comme $K_{l}$ en remplaçant $D$ par $D^{\prime}$. On peut vérifier que la méthode de Kazhdan [20] reprise dans [3] s'applique directement, en considérant $D$ comme "corps de base" : si l'entier $m$ est assez grand (en un sens ne dépendant que des entiers $r, l$ et de 
$D)$, alors (3.9) est un isomorphisme d'algèbres. Notons que, dans cette formulation, on ne suppose pas que $D$ et $D^{\prime}$ aient la même dimension ou le même invariant de Hasse, ni même que leurs centres soient proches.

3.6. Corps proches et représentations. - Soit un entier $l \geqslant 0$, et soit $m \geqslant 1$ un entier comme dans le théorème 3.8 , de sorte que $\zeta_{G_{F}, G_{L}}^{m, l}$ soit un isomorphisme d'algèbres. En utilisant la correspondance $(\pi, V) \mapsto V^{l}$ entre les classes d'isomorphisme de représentations irréductibles de $G_{F}$ ayant un vecteur non nul fixé par $K_{F}^{l}$ et les $\mathcal{H}_{F}^{l}$-modules simples, on obtient la proposition suivante.

Proposition 3.10. - L'isomorphisme d'algèbres $\zeta_{G_{F}, G_{L}}^{m, l}$ induit une bijection $\boldsymbol{\zeta}_{G_{F}, G_{L}}^{m, l}$ entre l'ensemble des classes d'isomorphisme de représentations irréductibles de $G_{F}$ de niveau inférieur ou égal à l (resp. égal à l) et l'ensemble des classes d'isomorphisme de représentations irréductibles de $G_{L}$ de niveau inférieur ou égal à l (resp. égal à l).

On note $U_{F}^{0}$ le groupe des unités de $\mathfrak{o}_{F}$ et, pour $k \geqslant 1$, on pose $U_{F}^{k}=1+\mathfrak{p}_{F}^{k}$, qui est un sous-groupe de $U_{F}^{0}$. En prenant $r=1$ et $D=F$ dans la proposition précédente, on voit que $\boldsymbol{\zeta}_{F^{\times}, L^{\times}}^{m, l}$ réalise une bijection entre l'ensemble des caractères de $F^{\times}$triviaux sur $U_{F}^{l}$ et l'ensemble des caractères de $L^{\times}$triviaux sur $U_{L}^{l}$. D'ailleurs on peut prendre $m=l$ et obtenir ce résultat directement : l'isomorphisme d'anneaux $\lambda_{F, L}^{m}$ induit un isomorphisme de groupes entre $U_{F}^{0} / U_{F}^{m}$ et $U_{L}^{0} / U_{L}^{m}$, qui se prolonge de manière unique en un isomorphisme de groupes :

$$
\lambda_{F, L}^{m, \times}: F^{\times} / U_{F}^{m} \rightarrow L^{\times} / U_{L}^{m}
$$

tel que les classes de $\varpi_{F}$ et $\varpi_{L}$ se correspondent. D'autre part, $\mathcal{H}_{F}^{m}$ s'identifie à l'algèbre du groupe $F^{\times} / U_{F}^{m}$ et $\mathcal{H}_{L}^{m}$ à l'algèbre du groupe $L^{\times} / U_{L}^{m}$, et (3.10) induit un isomorphisme d'algèbres entre $\mathcal{H}_{F}^{m}$ et $\mathcal{H}_{L}^{m}$ qui n'est autre que $\zeta_{F^{\times}, L^{\times}}^{m}$.

Comme le centre $Z_{F}$ de $G_{F}$ s'identifie naturellement à $F^{\times}$, on a le lemme suivant.

Lemme 3.11. - Soit $\pi$ une représentation irréductible de $G_{F}$ de niveau inférieur à $l$. Le caractère central $\omega$ de $\pi$ est trivial sur $U_{F}^{l}$, et celui de $\boldsymbol{\zeta}_{G_{F}, G_{L}}^{m, l}(\pi)$ est $\boldsymbol{\zeta}_{F^{\times}, L^{\times}}^{m, l}(\omega)$.

Soit $\psi_{F}$ un caractère additif de $F$ de conducteur $\mathfrak{o}_{F}$, c'est-à-dire trivial sur $\mathfrak{p}_{F}$ mais pas sur $\mathfrak{o}_{F}$. Pour toute représentation irréductible $\pi$ de $G_{F}$, Godement et Jacquet [18] ont défini des facteurs $\varepsilon\left(s, \pi, \psi_{F}\right)$ et $\varepsilon^{\prime}\left(s, \pi, \psi_{F}\right)$. On sait que le facteur $\varepsilon\left(s, \pi, \psi_{F}\right)$ est égal à $c q^{-m(\pi) s}$, où $c$ est une constante non nulle et $q$ le cardinal du corps résiduel de $F$ (qui est aussi celui du corps résiduel de $L$ ). L'entier $m(\pi)$ ne dépend que de la classe 
d'ismorphisme de $\pi$, et pas du choix du caractère $\psi_{F}$ de conducteur $\mathfrak{o}_{F}$. Soit maintenant $\psi_{L}$ un caractère additif de $L$ de conducteur $\mathfrak{o}_{L}$ vérifiant :

$$
\psi_{F}\left(\varpi_{F}^{-m} x\right)=\psi_{L}\left(\varpi_{L}^{-m} y\right)
$$

pour tout $x \in \mathfrak{o}_{F}$ et tout $y \in \mathfrak{o}_{L}$ dont les classes se correspondent par $\lambda_{F, L}^{m}$. La proposition suivante est montrée par exemple dans [3, théorèmes 2.17 et 2.19].

Proposition 3.12. - Soit $\pi$ une représentation irréductible de $G_{F}$ de niveau inférieur $\grave{a}$ l, et posons $\tau=\boldsymbol{\zeta}_{G_{F}, G_{L}}^{m, l}(\pi)$. Si m est comme dans le théorème 3.8, alors :

(1) $\pi$ est de carré intégrable (resp. cuspidale) si et seulement si $\tau$ l'est;

(2) on a $\varepsilon^{\prime}\left(s, \pi, \psi_{F}\right)=\varepsilon^{\prime}\left(s, \tau, \psi_{L}\right)$;

(3) si $\pi$ est cuspidale, on a $\varepsilon\left(s, \pi, \psi_{F}\right)=\varepsilon\left(s, \tau, \psi_{L}\right)$ et $m(\pi)=m(\tau)$.

Remarque 3.13. - Comme on l'a dit plus haut, si $D$ est égale à $F$ et si $l \geqslant 1$, Lemaire montre dans $[\mathbf{2 1}]$ qu'on peut prendre $m=l$ dans le théorème 3.8 (pour $D$ quelconque, ce résultat effectif sur $m$ est conjectural). Il y montre également que si $m=l+1$ et si $\pi$ est générique, on a $m(\pi)=m(\tau)$.

3.7. Corps proches et correspondance de Jacquet-Langlands. - On note $H_{F}$ le groupe $\mathrm{GL}_{r d}(F)$ et $\mathbf{J L}_{F}$ la correspondance de Jacquet-Langlands entre $H_{F}$ et $G_{F}$, et on emploie des notations analogues pour $L$. Soit $\sigma$ une représentation de carré intégrable de $G_{F}$. D'après $\left[\mathbf{3}\right.$, lemme 5.12], l'ensemble $X\left(G_{F}, \sigma\right)$ des classes d'isomorphisme de représentations de carré intégrable $\pi$ de $G_{F}$, de même caractère central et de même facteur $\varepsilon^{\prime}$ que ceux de $\sigma$, est fini. On note $l^{\prime}(\sigma)$ le plus grand des $l(\pi)$ pour $\pi \in \mathcal{X}\left(G_{F}, \sigma\right)$.

Proposition 3.14. - Soit $\sigma$ une représentation de carré intégrable de $H_{F}$, et soit un entier $l \geqslant 0$ supérieur à $l^{\prime}(\sigma)$ et $l^{\prime}\left(\mathbf{J L}_{F}(\sigma)\right)$. Si $m$ est assez grand, on a :

$$
\mathbf{J L}_{L} \circ \boldsymbol{\zeta}_{H_{F}, H_{L}}^{m, l}(\sigma)=\boldsymbol{\zeta}_{G_{F}, G_{L}}^{m, l} \circ \mathbf{J L}_{F}(\sigma)
$$

Démonstration. - La représentation $\sigma^{\prime}=\mathbf{J L}_{F}(\sigma)$ est une représentation de carré intégrable de $G_{F}$. Si $m$ est comme dans la proposition 3.12, les représentations $\tau=\boldsymbol{\zeta}_{H_{F}, H_{L}}^{m, l}(\sigma)$ et $\boldsymbol{\zeta}_{G_{F}, G_{L}}^{m, l}\left(\sigma^{\prime}\right)$ sont bien définies et de carré intégrable, et la représentation $\tau^{\prime}=\mathbf{J L}_{L}(\tau)$ est de carré intégrable. D'après [3, théorème 5.1], la correspondance de Jacquet-Langlands préserve le caractère central et le facteur $\varepsilon^{\prime}$. Aussi la proposition 3.12 implique-t-elle que $\boldsymbol{\zeta}_{G_{F}, G_{L}}^{m, l}$ et $\mathbf{J L}_{L} \circ \boldsymbol{\zeta}_{H_{F}, H_{L}}^{m, l} \circ \mathbf{J L}_{F}^{-1}$ réalisent deux bijections de $\mathcal{X}\left(G_{F}, \sigma^{\prime}\right)$ sur $\mathcal{X}\left(G_{L}, \tau^{\prime}\right)$. 
D'après les relations d'orthogonalité des caractères sur $G_{F}$ (voir [3, corollaire 5.13]), deux représentations de carré intégrable de $G_{F}$ sont non isomorphes si et seulement s'il existe un élément semisimple régulier elliptique de $G_{F}$ sur lequel leurs fonctions-caractères diffèrent. Puisque $X\left(G_{F}, \sigma^{\prime}\right)$ est fini, la proposition 5.11 de [3] implique que, si $m$ est assez grand, les représentations $\tau^{\prime}$ et $\boldsymbol{\zeta}_{G_{F}, G_{L}}^{m, l}\left(\pi^{\prime}\right)$ diffèrent pour toute représentation $\pi^{\prime}$ dans $X\left(G_{F}, \sigma^{\prime}\right)$ distincte de $\sigma^{\prime}$. Par élimination, on en déduit que $\tau^{\prime}=\boldsymbol{\zeta}_{G_{F}, G_{L}}^{m, l}\left(\sigma^{\prime}\right)$.

3.8. Corps proches et induction parabolique. - On suppose maintenant que $l \geqslant 1$. On note $\mathcal{R}^{l}\left(G_{F}\right)$ la sous-catégorie pleine de $\mathcal{R}\left(G_{F}\right)$ dont les objets sont les représentations engendrées par leurs vecteurs fixes sous $K_{F}^{l}$. Ce groupe admet, pour chaque sous-groupe de Levi standard $M_{F, \lambda}$ de $G_{F}$, la décomposition triangulaire :

$$
K_{F}^{l}=\left(\bar{U}_{F, \lambda} \cap K_{F}^{l}\right) \cdot\left(M_{F, \lambda} \cap K_{F}^{l}\right) \cdot\left(U_{F, \lambda} \cap K_{F}^{l}\right),
$$

où $U_{F, \lambda}$ désigne le radical unipotent de $P_{F, \lambda}$ et $\bar{U}_{F, \lambda}$ celui du sous-groupe parabolique de $G_{F}$ opposé à $P_{F, \lambda}$ par rapport à $M_{F, \lambda}$. Puisque $K_{F}^{l}$ satisfait aux conditions 3.7 .1 et 3.7.2 de [10], la catégorie $\mathcal{R}^{l}\left(G_{F}\right)$ est stable par sous-quotients et le foncteur $(\pi, V) \mapsto V^{l}$ définit une équivalence de catégories entre $\mathcal{R}^{l}\left(G_{F}\right)$ et la catégorie des $\mathcal{H}_{F}^{l}$-modules à gauche (voir [10, corollaire 3.9]). L'isomorphisme d'algèbres $\zeta_{G_{F}, G_{L}}^{m, l}$ permet donc de définir une équivalence de catégories, que l'on note $\boldsymbol{\zeta}_{G_{F}, G_{L}}^{m, l}$, entre $\mathcal{R}^{l}\left(G_{F}\right)$ et $\mathcal{R}^{l}\left(G_{L}\right)$. Cette équivalence de catégories induit, par restriction aux représentations irréductibles, la bijection de la proposition 3.10 .

On fixe une partition $\lambda$ de l'entier $r$ comme en (2.1), et on pose $M_{F}=M_{F, \lambda}, P_{F}=P_{F, \lambda}$ et $K_{F, \lambda}^{l}=K_{F}^{l} \cap M_{F}$. En remplaçant $G_{F}$ par $M_{F}$ et $K_{F}^{l}$ par $K_{F, \lambda}^{l}$, on définit comme plus haut la sous-catégorie pleine $\mathcal{R}^{l}\left(M_{F}\right)$ de $\mathcal{R}\left(M_{F}\right)$. On note :

$$
\iota_{P_{F}}^{G_{F}}: \mathcal{R}\left(M_{F}\right) \rightarrow \mathcal{R}\left(G_{F}\right)
$$

le foncteur d'induction parabolique normalisée de $M_{F}$ à $G_{F}$ suivant $P_{F}$, et :

$$
\boldsymbol{r}_{P_{F}}^{G_{F}}: \mathcal{R}\left(G_{F}\right) \rightarrow \mathcal{R}\left(M_{F}\right)
$$

le foncteur de restriction de Jacquet normalisée de $G_{F}$ à $M_{F}$ suivant $P_{F}$. D'après la démonstration de [13, proposition 4], le foncteur $\boldsymbol{\iota}_{P_{F}}^{G_{F}}$ envoie $\mathcal{R}^{l}\left(M_{F}\right)$ dans $\mathcal{R}^{l}\left(G_{F}\right)$ et le foncteur $\boldsymbol{r}_{P_{F}}^{G_{F}}$ envoie $\mathcal{R}^{l}\left(G_{F}\right)$ dans $\mathcal{R}^{l}\left(M_{F}\right)$. D'autre part, l'identification du sous-groupe de Levi $M_{F}$ au produit $G_{F, r_{1}} \times \cdots \times G_{F, r_{k}}$ induit une identification :

$$
\mathcal{H}\left(M_{F}, K_{F, \lambda}^{l}\right)=\mathcal{H}_{F, r_{1}}^{l} \otimes \cdots \otimes \mathcal{H}_{F, r_{k}}^{l} .
$$


Pourvu que $m$ soit assez grand, l'isomorphisme $\lambda_{F, L}^{m}$ et les uniformisantes $\varpi_{D_{F}}$ et $\varpi_{D_{L}}$ permettent de construire, grâce au théorème 3.8, pour chaque $i \in\{1, \ldots, k\}$, un isomorphisme d'algèbres $\zeta_{G_{F, r_{i}}, G_{L, r_{i}}}^{m, l}$ de $\mathcal{H}_{F, r_{i}}^{l}$ dans $\mathcal{H}_{L, r_{i}}^{l}$. On en tire isomorphisme d'algèbres :

$$
\zeta_{M_{F}, M_{L}}^{m, l}=\bigotimes_{i=1}^{k} \zeta_{G_{F, r_{i}}, G_{L, r_{i}}}^{m, l}: \mathcal{H}\left(M_{F}, K_{F, \lambda}^{l}\right) \rightarrow \mathcal{H}\left(M_{L}, K_{L, \lambda}^{l}\right)
$$

qui permet de définir comme plus haut une équivalence de catégorie $\boldsymbol{\zeta}_{M_{F}, M_{L}}^{m, l}$ entre $\mathcal{R}^{l}\left(M_{F}\right)$ et $\mathcal{R}^{l}\left(M_{L}\right)$.

Dans le cas où $D$ est égale à $F$, le résultat suivant est une variante de la proposition $[\mathbf{1 9}$, A.4.1] dans laquelle on utilise les sous-groupes de congruence d'un sous-groupe d'Iwahori au lieu des sous-groupes de congruence d'un sous-groupe compact maximal.

Proposition 3.15. - On suppose que $m$ est assez grand pour que les équivalences de catégories $\boldsymbol{\zeta}_{G_{F}, G_{L}}^{m, l}$ et $\boldsymbol{\zeta}_{M_{F}, M_{L}}^{m, l}$ soient bien définies. Alors, pour toute représentation $\sigma$ dans $\mathcal{R}^{l}\left(M_{F}\right)$, on $a$ :

$$
\boldsymbol{\iota}_{P_{L}}^{G_{L}} \circ \boldsymbol{\zeta}_{M_{F}, M_{L}}^{m, l}(\sigma)=\boldsymbol{\zeta}_{G_{F}, G_{L}}^{m, l} \circ \boldsymbol{\iota}_{P_{F}}^{G_{F}}(\sigma),
$$

et pour toute représentation $\pi$ dans $\mathcal{R}^{l}\left(G_{F}\right)$, on a :

$$
\boldsymbol{r}_{P_{L}}^{G_{L}} \circ \boldsymbol{\zeta}_{G_{F}, G_{L}}^{m, l}(\pi)=\boldsymbol{\zeta}_{M_{F}, M_{L}}^{m, l} \circ \boldsymbol{r}_{P_{F}}^{G_{F}}(\pi)
$$

Démonstration. - La preuve est analogue à celle de [19, proposition A.4.1]. Cette dernière est en effet basée sur les résultats de Bushnell [13], qui sont valables pour n'importe quel groupe réductif connexe. Notons pour commencer que remplacer les sousgroupes de congruence d'un sous-groupe d'Iwahori par les sous-groupes de congruence d'un sous-groupe compact maximal ne change rien à l'affaire. D'ailleurs dans [13], ce sont ces derniers que Bushnell utilise. Notons $Z_{F, \lambda}$ le centre de $M_{F}$, identifié au produit de $k$ copies de $F^{\times}$, et $Z_{F, \lambda}^{++}$le sous-ensemble de $Z_{F, \lambda}$ formé des éléments $\left(x_{1}, \ldots, x_{k}\right)$ tels qu'on ait $v_{F}\left(x_{i}\right)>v_{F}\left(x_{i+1}\right)$ pour tout entier $1 \leqslant i \leqslant k-1$, où $v_{F}$ désigne la valuation normalisée de $F$. Notons aussi $M_{F}^{+}$le sous-ensemble de $M_{F}$ formé des éléments $\left(P_{F}, K_{F, r}^{l}\right)$-positifs au sens de [19, A.3]. Alors $Z_{F, \lambda}^{++}$est contenu dans $M_{F}^{+}$, et $M_{F}^{+}$est bi-invariant par $K_{F, r}^{l}$. On procède alors exactement comme dans la démonstration de [19, A.3], en remarquant que, par construction, $\zeta_{M_{F}, M_{L}}^{m, l}$ envoie toute fonction à support dans $K_{F, \lambda}^{l} Z_{F, \lambda}^{++} K_{F, \lambda}^{l}=K_{F, \lambda}^{l} Z_{F, \lambda}^{++}$sur une fonction à support dans $K_{L, \lambda}^{l} Z_{L, \lambda}^{++}$, et toute fonction à support dans $K_{F, \lambda}^{l} M_{F}^{+} K_{F, \lambda}^{l}$ sur une fonction à support dans $K_{L, \lambda}^{l} M_{L}^{+} K_{L, \lambda}^{l}$. 
Notons que, dans le présent article, nous n'utilisons pas la compatibilité par rapport aux foncteurs de Jacquet.

Corollaire 3.16. - Soit $\pi$ une représentation irréductible de $G_{F}$ de la forme:

$$
L\left(\nu_{F}^{b_{1}} \pi_{1}, \ldots, \nu_{F}^{b_{k}} \pi_{k}\right)
$$

où les $\pi_{i}$ sont des représentations de carré intégrable de niveau inférieur ou égal à $l$ et les $b_{i}$ des entiers tels que $b_{1} \geqslant \cdots \geqslant b_{k}$. Si m est comme dans la proposition 3.15, on a:

$$
\boldsymbol{\zeta}_{G_{F}, G_{L}}^{m, l}(\pi)=L\left(\nu_{L}^{b_{1}} \boldsymbol{\zeta}_{G_{F, r_{1}}, G_{L, r_{1}}}^{m, l}\left(\pi_{1}\right), \ldots, \nu_{L}^{b_{k}} \boldsymbol{\zeta}_{G_{F, r_{k}}, G_{L, r_{k}}}^{m, l}\left(\pi_{k}\right)\right) .
$$

Démonstration. - Si $m$ est comme dans la proposition 3.15, on a :

$$
\boldsymbol{\zeta}_{G_{F}, G_{L}}^{m, l}\left(\nu_{F}^{b_{1}} \pi_{1} \times \cdots \times \nu_{F}^{b_{k}} \pi_{k}\right)=\nu_{L}^{b_{1}} \boldsymbol{\zeta}_{G_{F, r_{1}}, G_{L, r_{1}}}^{m, l}\left(\pi_{1}\right) \times \cdots \times \nu_{L}^{b_{k}} \boldsymbol{\zeta}_{G_{F, r_{k}}, G_{L, r_{k}}}^{m, l}\left(\pi_{k}\right) .
$$

Pour chaque entier $i \in\{1, \ldots, k\}$, la représentation $\tau_{i}=\boldsymbol{\zeta}_{G_{F, r_{i}}, G_{L, r_{i}}}^{m, l}\left(\pi_{i}\right)$ est irréductible et de carré intégrable d'après la proposition 3.12, et le membre de droite de (3.14) est bien défini. Comme la catégorie $\mathcal{R}^{l}\left(G_{F}\right)$ est stable par sous-quotient et que l'équivalence de catégories $\boldsymbol{\zeta}_{G_{F}, G_{L}}^{m, l}$ envoie toute suite exacte dans $\mathcal{R}^{l}\left(G_{F}\right)$ sur une suite exacte dans $\mathcal{R}^{l}\left(G_{L}\right)$, la représentation $\boldsymbol{\zeta}_{G_{F}, G_{L}}^{m, l}(\pi)$ est bien définie et $\boldsymbol{\zeta}_{G_{F}, G_{L}}^{m, l}(\pi)=L\left(\nu_{L}^{b_{1}} \tau_{1}, \ldots, \nu_{L}^{b_{k}} \tau_{k}\right)$.

Proposition 3.17. - Soit $\sigma$ une représentation de carré intégrable de $G_{F}$ de la forme $\sigma=T(\rho, k)$, où $\rho$ est une représentation cuspidale unitaire de $G_{F, n}$ et $k$ un entier tel que $r=k n$. On suppose que $\sigma$ et $\rho$ sont de niveau inférieur à $l$. Si $m$ est comme dans la proposition 3.15, on a $\boldsymbol{\zeta}_{G_{F}, G_{L}}^{m, l}(\sigma)=T\left(\boldsymbol{\zeta}_{G_{F, n}, G_{L, n}}^{m, l}(\rho), k\right)$ et $s\left(\boldsymbol{\zeta}_{G_{F}, G_{L}}^{m, l}(\sigma)\right)=s(\sigma)$.

Démonstration. - Pour $k=1$, c'est une conséquence des propositions 3.12 et 3.14. Supposons que $k \geqslant 2$ et définissons des entiers $a_{i}$ par (1.1). Alors $\sigma=T(\rho, k)$ est l'unique sous-représentation irréductible de $\nu_{F}^{s(\sigma) a_{1}} \rho \times \cdots \times \nu_{F}^{s(\sigma) a_{k}} \rho$. Le résultat est alors une conséquence de la proposition 3.15.

3.9. Preuve de U1. - On a maintenant réuni tous les ingrédients nécessaires à la preuve du théorème suivant, qui est le résultat principal de ce chapitre.

Théorème 3.18. - Si F est de caractéristique non nulle, alors U1 est vraie.

Démonstration. - Soient $k, n \geqslant 1$ des entiers, soit $\sigma$ une représentation de carré intégrable de $G_{F, n}$, soit $\pi=u(\sigma, k)$ et soit $V$ l'espace de $\pi$. D'après [15, proposition 3.1.2], la représentation $\pi$ est hermitienne. Soit donc $h$ une forme hermitienne non dégénérée sur $V$ invariante par $\pi$. Quitte à multiplier $h$ par un scalaire, on peut supposer que $h$ prend 
des valeurs strictement positives. Plus précisément, on fixe un vecteur non nul $v_{0} \in V$ et on suppose que $h\left(v_{0}, v_{0}\right)>0$. On va montrer que $h$ est définie positive, c'est-à-dire que, pour tout $v \in V$ non nul, on a $h(v, v)>0$.

Soit $v \in V$ un vecteur non nul, et soit un entier $l \geqslant 1$ assez grand pour que $v, v_{0} \in V^{l}$. Soit un entier $m \geqslant l$ comme dans la proposition 3.15. Fixons un corps commutatif localement compact non archimédien de caractéristique nulle $L$ qui soit $m$-proche de $F$, une uniformisante $\varpi_{L}$ de $L$ et un isomorphisme (3.2) faisant se correspondre les classes de $\varpi_{F}$ et $\varpi_{L}$. C'est toujours possible puisque $F$ est de caractéristique non nulle. On fixe une $L$-algèbre à division $D_{L}$ de centre $L$ ayant la même la même dimension et le même invariant de Hasse que $D_{F}$, et une uniformisante $\varpi_{D_{L}}$ telle que $\varpi_{D_{L}}^{d}=\varpi_{L}$. On construit comme au paragraphe 3.5 un isomorphisme d'algèbres tel que le corollaire 3.16 et la proposition 3.17 soient vérifiés. Alors d'après le corollaire 3.16 , on a :

$$
\boldsymbol{\zeta}_{G_{F}, G_{L}}^{m, l}(u(\sigma, k))=u\left(\boldsymbol{\zeta}_{G_{F, n}, G_{L, n}}^{m, l}(\sigma), k\right) .
$$

Notons $\tau$ le membre de droite de cette égalité et $W$ l'espace de $\tau$. Nous savons que la représentation $\tau$ est unitaire, car $L$ est de caractéristique nulle. Soit $f$ un produit scalaire hermitien sur $W$ stable par $\tau$. Puisque les $\mathcal{H}_{F}^{l}$-modules $V^{l}$ et $W^{l}$ sont isomorphes $\left(\mathcal{H}_{F}^{l}\right.$ agissant sur $W^{l}$ via $\zeta_{G_{F}, G_{L}}^{m, l}$ ), la forme $h^{l}$ définit par transport de structure une forme hermitienne non dégénérée $\varphi$ sur $W^{l}$ vérifiant la relation (3.1) et prenant des valeurs strictement positives. D'après la proposition 3.5 , la forme hermitienne $\varphi$ est proportionnelle à $f^{l}$. Puisque $f^{l}$ est définie positive et que $\varphi$ prend des valeurs strictement positives, la constante de proportionnalité est strictement positive, et donc $h(v, v)=h^{l}(v, v)>0$.

Remarque 3.19. - Reprenons les hypothèses et les notations du paragraphe 3.8 (en particulier, on a $m \geqslant l \geqslant 1$ ). D'après le théorème de classification 2.6 , le théorème 3.18 (et sa démonstration) et les résultats du paragraphe 3.8, on obtient a posteriori que si $m$ est comme dans la proposition 3.15, alors l'application $\zeta_{G_{F}, G_{L}}^{m, l}$ induit une bijection entre les classes d'isomorphisme de représentations irréductibles unitaires de $G_{F}$ ayant un vecteur non nul fixé par $K_{F}^{l}$, et l'ensemble des classes d'isomorphisme de représentations irréductibles unitaires de $G_{L}$ ayant un vecteur non nul fixé par $K_{L}^{l}$.

\section{Une seconde preuve de U1 en caractéristique non nulle}

4.1. La méthode de Tadić. - Comme on l'a dit au paragraphe 2.5, connaissant U0, il existe une seconde manière de prouver U1 (voir le paragraphe 2.5 pour l'énoncé de U) : 
Proposition 4.1. - Si U0 et U sont vraies, alors U1 l'est aussi.

Démonstration. - Supposons que l'on a prouvé U0 et U. On veut montrer que pour tous entiers $k, n \geqslant 1$ et toute représentation de carré intégrable $\sigma$ de $G_{n}$, la représentation $u(\sigma, k)$ est unitaire. La démonstration se fait par récurrence sur $k$. Pour $k=1$, la représentation $u(\sigma, k)=\sigma$ est unitaire. Ensuite, on utilise le lemme suivant.

Lemme 4.2. - Soit $\pi$ une representation irréductible unitaire de $G_{n}$, et soit $\alpha$ le plus petit nombre réel $\geqslant 0$ tel que $\nu^{\alpha} \pi \times \nu^{-\alpha} \pi$ soit réductible. Alors pour tout $0 \leqslant \beta \leqslant \alpha$, les sous-quotients irréductibles de $\nu^{\beta} \pi \times \nu^{-\beta} \pi$ sont unitaires.

Ce lemme est expliqué dans $[\mathbf{3 2}, \S 3(\mathrm{c})]$ où Tadić l'attribue à Milicić. Notons que, puisque U0 est vraie, $\alpha$ est non nul. Posons $s=s(\sigma)$ et appliquons le lemme 4.2 à $\sigma$. D'après U0 et la remarque 2.5, la représentation $\nu^{s \alpha} \sigma \times \nu^{-s \alpha} \sigma$ est irréductible pour tout nombre réel $0 \leqslant \alpha<1 / 2$. On en déduit que la représentation $u(\sigma, 2)$ est unitaire, puisque c'est un sous-quotient irréductible de $\nu^{s / 2} \sigma \times \nu^{-s / 2} \sigma$. Fixons un entier $k \geqslant 2$ et supposons que la représentation $u(\sigma, k)$ est unitaire. D'après $(2.7)$, on a :

$$
\begin{aligned}
u(\sigma, k+1) & =L\left(\nu^{s k / 2} \sigma, \nu^{s(k / 2-1)} \sigma, \ldots, \nu^{-s k / 2} \sigma\right), \\
u(\sigma, k-1) & =L\left(\nu^{s(k / 2-1)} \sigma, \nu^{s(k / 2-2)} \sigma, \ldots, \nu^{-s(k / 2-1)} \sigma\right), \\
\nu^{s / 2} u(\sigma, k) & =L\left(\nu^{s k / 2} \sigma, \nu^{s(k / 2-1)} \sigma, \ldots, \nu^{-s(k / 2-1)} \sigma\right), \\
\nu^{-s / 2} u(\sigma, k) & =L\left(\nu^{s(k / 2-1)} \sigma, \nu^{s(k / 2-2)} \sigma, \ldots, \nu^{-s k / 2} \sigma\right) .
\end{aligned}
$$

On définit les ensembles suivants de classes d'isomorphisme de représentations essentiellement de carré intégrable de $G_{n}$ :

$$
\begin{aligned}
\delta & =\left\{\left[\nu^{s i} \sigma\right] \mid-k / 2 \leqslant i \leqslant k / 2\right\}, \\
\delta^{\prime} & =\left\{\left[\nu^{s i} \sigma\right] \mid 1-k / 2 \leqslant i \leqslant k / 2-1\right\}, \\
\delta_{1} & =\left\{\left[\nu^{s i} \sigma\right] \mid 1-k / 2 \leqslant i \leqslant k / 2\right\}, \\
\delta_{2} & =\left\{\left[\nu^{s i} \sigma\right] \mid-k / 2 \leqslant i \leqslant k / 2-1\right\} .
\end{aligned}
$$

Puisque $\mathrm{U}$ est vraie, la représentation $u(\sigma, k+1) \times u(\sigma, k-1)$ est irréductible et de classe égale à $\mathcal{T}\left(\delta+\delta^{\prime}\right)$. D'autre part, comme $\delta+\delta^{\prime}=\delta_{1}+\delta_{2}$, c'est un sous-quotient irréductible de $\nu^{s / 2} u(\sigma, k) \times \nu^{-s / 2} u(\sigma, k)$. Puisque $u(\sigma, k)$ est unitaire, $\nu^{s \alpha} u(\sigma, k) \times \nu^{-s \alpha} u(\sigma, k)$ est irréductible pour tout nombre réel $0 \leqslant \alpha<1 / 2$, d'après U0 et la remarque 2.5. On en déduit que $u(\sigma, k+1) \times u(\sigma, k-1)$ est unitaire grâce au lemme 4.2. Or on sait que si le produit de deux représentations irréductibles hermitiennes est irréductible et unitaire, 
alors ces deux représentations sont unitaires d'après $[\mathbf{3 2}, \S 3(\mathrm{~d})]$ (voir aussi $[\mathbf{2 8}$, Lemma $1.2])$. Donc $u(\sigma, k+1)$ est unitaire, ce qui achève la démonstration de la proposition.

Remarque 4.3. - L'avantage de cette approche de Tadić est qu'elle permet de prouver U1 sans utiliser d'argument global. Remarquons qu'à ce stade de l'article, grâce aux résultats du paragraphe 3.8, on a une preuve immédiate de $\mathrm{U}$, au moyen de la méthode des corps proches qui permet de se ramener au cas où $F$ est de caractéristique nulle, auquel cas U est une conséquence de U0 et U1. Dans les numéros suivants, nous donnons une preuve de U, qui nous a été suggérée par Tadić, s'appuyant sur la théorie des types comme dans [28]. Cette approche permet de se ramener au cas où $D$ est égale à $F$, auquel cas U a été prouvé par Tadić dans [30].

4.2. Une preuve de $\mathbf{U}$ par la théorie des types. - Soient des entiers $n \geqslant 1$ et $k \geqslant 2$, et soit $\sigma$ une représentation de carré intégrable de $G_{n}$. On veut montrer que la représentation :

$$
u(\sigma, k+1) \times u(\sigma, k-1)
$$

de $G_{2 k n}$ est irréductible. D'après la propriété P2 (voir le paragraphe 2.2), il existe un diviseur $a$ de $n$ et une représentation irréductible cuspidale unitaire $\rho$ de $G_{a}$ tels que $\sigma$ soit isomorphe à $T(\rho, b)$, où l'entier $b$ est défini par $n=a b$. On va montrer qu'à la représentation cuspidale $\rho$ correspond une extension finie $K$ de $F$ telle que, si l'on note $1_{K^{\times}}$le caractère trivial de $K^{\times}$et $\sigma^{\prime}$ la représentation de carré intégrable $T\left(1_{K^{\times}}, b\right)$, le produit (4.1) est une représentation irréductible de $G_{2 k n}$ si et seulement si :

$$
u\left(\sigma^{\prime}, k+1\right) \times u\left(\sigma^{\prime}, k-1\right)
$$

est une représentation irréductible de $\mathrm{GL}_{2 k b}(K)$, ce qui a été prouvé par Tadić dans [30].

4.3. Soient $a \geqslant 1$ un entier et $\rho$ une représentation cuspidale de $G_{a}$. Pour tout entier $n \geqslant 1$, on note $\mathcal{R}_{n}^{\rho}$ la sous-catégorie pleine de $\mathcal{R}_{a n}$ constituée des représentations dont tous les facteurs de composition sont des sous-quotients d'induites paraboliques normalisées de $(\rho \otimes \cdots \otimes \rho) \chi$ à $G_{a n}$, où $\chi$ est un caractère non ramifié de $G_{a} \times \cdots \times G_{a}$. On note $\mathcal{G}_{n}^{\rho}$ le groupe de Grothendieck des représentations de longueur finie de $\mathcal{R}_{n}^{\rho}$ (c'est un sous-groupe de $\left.\mathcal{G}_{a n}\right)$ et $\mathcal{J}_{n}^{\rho}$ l'ensemble des classes d'isomorphisme de ses représentations irréductibles, de sorte que $\mathcal{G}_{n}^{\rho}$ est un $\mathbb{Z}$-module libre de base $\mathcal{J}_{n}^{\rho}$. Il est commode de convenir que $\mathcal{G}_{0}^{\rho}=\mathbb{Z}$ 
et $\mathcal{R}_{0}^{\rho}=\{0\}$. Soit :

$$
\mathcal{G}^{\rho}=\bigoplus_{n \geqslant 0} \mathcal{G}_{n}^{\rho}
$$

C'est un $\mathbb{Z}$-module libre gradué de base :

$$
\mathcal{J}^{\rho}=\bigcup_{n \geqslant 0} \mathcal{J}_{n}^{\rho}
$$

L'application $(\sigma, \tau) \mapsto \sigma \times \tau$ définie au paragraphe 2.2 induit une application $\mathbb{Z}$-bilinéaire de $\mathcal{G}^{\rho} \times \mathcal{G}^{\rho}$ dans $\mathcal{G}^{\rho}$ faisant de $\mathcal{G}^{\rho}$ une $\mathbb{Z}$-algèbre graduée, associative et commutative (voir $[35, \S 1]$ et la propriété P1 du paragraphe 2.2).

On fixe maintenant un type simple $(J, j)$ contenu dans $\rho$ (pour la notion de type simple et les quantités qui lui seront attachées, on renvoie à [27] ou à [28, §2.6-2.7]). Pour chaque entier $n \geqslant 1$, il lui correspond via [27] un type $\left(J_{n}, j_{n}\right)$ pour le bloc $\mathcal{R}_{n}^{\rho}$ de $\mathcal{R}_{a n}$ qui est une paire couvrante de la paire $\left(J^{n}, j^{\otimes n}\right)$ du sous-groupe de Levi standard $M_{\lambda}$ de $G_{a n}$ relativement à $P_{\lambda}$. On note $\mathcal{H}_{n}^{j}$ l'algèbre de Hecke de $G_{a n}$ relative à la paire $\left(J_{n}, j_{n}\right)$ et $\operatorname{Mod}\left(\mathcal{H}_{n}^{j}\right)$ la catégorie des $\mathcal{H}_{n}^{j}$-modules à droite. Le foncteur :

$$
\mathbf{M}_{n}^{j}: \pi \mapsto \operatorname{Hom}_{J_{n}}\left(j_{n}, \pi\right)
$$

est une équivalence de catégories de $\mathcal{R}_{n}^{\rho}$ vers $\operatorname{Mod}\left(\mathcal{H}_{n}^{j}\right)$. Pour tous entiers $m, n \geqslant 1$, on a un homomorphisme injectif de $\mathbb{C}$-algèbres :

$$
t_{m, n}^{j}: \mathcal{H}_{m}^{j} \otimes \mathcal{H}_{n}^{j} \rightarrow \mathcal{H}_{m+n}^{j}
$$

faisant de $\mathcal{H}_{m+n}^{j}$ un module à droite sur $\mathcal{H}_{m}^{j} \otimes \mathcal{H}_{n}^{j}$, et définissant un foncteur :

$$
\boldsymbol{t}_{m, n}^{j}: M \mapsto \operatorname{Hom}_{\mathcal{H}_{m}^{j} \otimes \mathcal{H}_{n}^{j}}\left(\mathcal{H}_{m+n}^{j}, M\right)
$$

de la catégorie $\operatorname{Mod}\left(\mathcal{H}_{m}^{j} \otimes \mathcal{H}_{n}^{j}\right)$ dans la catégorie $\operatorname{Mod}\left(\mathcal{H}_{m+n}^{j}\right)$. On a le fait suivant, qui est l'un des résultats principaux de [14].

Fait 1 Soient $m, n \geqslant 1$ deux entiers et $\sigma, \tau$ des représentations respectivement dans $\mathcal{R}_{m}^{\rho}$ et $\mathcal{R}_{n}^{\rho}$. On a un isomorphisme canonique de $\mathcal{H}_{m+n}^{j}$-modules :

$$
\mathbf{M}_{m+n}^{j}(\sigma \times \tau) \simeq \boldsymbol{t}_{m, n}^{j}\left(\mathbf{M}_{m}^{j}(\sigma) \otimes \mathbf{M}_{n}^{j}(\tau)\right) .
$$

Remarque 4.4. - Un cas particulier remarquable de cette situation est donnée par le choix pour $\rho$ du caractère trivial de $F^{\times}=\mathrm{GL}_{1}(F)$, à quoi on associe le type simple constitué du sous-groupe compact maximal $U_{F}^{0}$ de $F^{\times}$et de son caractère trivial $1_{U_{F}^{0}}$. Alors pour chaque entier $n \geqslant 1$, le groupe $J_{n}$ est le sous-groupe d'Iwahori standard de 
$G_{n}$ et $j_{n}$ est son caractère trivial. Dans ce cas, on remplace les exposants $\rho$ et $j$ par un exposant $F$ dans les notations précédentes : par exemple, l'algèbre de Hecke $\mathcal{H}_{n}^{j}$ est notée $\mathcal{H}_{n}^{F}$ et la catégorie $\mathcal{R}_{n}^{\rho}$ est notée $\mathcal{R}_{n}^{F}$.

Soit $N$ le normalisateur de $j$ dans $G_{a}$. D'après [27] (voir aussi [28, Proposition 2.3]), il existe une extension finie $K$ de $F$ contenue dans l'algèbre de matrices $\mathrm{M}_{a}(D)$ telle que $N=K^{\times} J$. En outre, si l'on fixe une uniformisante $\varpi_{K}$ de $K$, alors $N$ est engendré par $J$ et $\varpi_{K}$. On note $\tilde{\jmath}$ l'unique prolongement de $j$ à $N$ tel que $\rho$ soit isomorphe à l'induite compacte de $\tilde{\jmath}$ à $G_{a}$. On note $\Psi_{1}$ l'unique isomorphisme de $\mathbb{C}$-algèbres de $\mathcal{H}_{1}^{K}$ dans $\mathcal{H}_{1}^{j}$ tel que la classe $\varpi_{K} U_{K}^{0}$ ait pour image la fonction de support $J \varpi_{K} J$ prenant en $\varpi_{K}$ la valeur $\widetilde{\jmath}\left(\varpi_{K}\right)$. On a le fait suivant, dont on trouvera une démonstration dans [28].

Fait 2 Il existe une famille d'isomorphismes de $\mathbb{C}$-algèbres :

$$
\Psi_{n}: \mathcal{H}_{n}^{K} \rightarrow \mathcal{H}_{n}^{j}, \quad n \geqslant 1
$$

tel que, pour tous $m, n \geqslant 1$, on ait $\Psi_{m+n} \circ t_{m, n}^{K}=t_{m, n}^{j} \circ\left(\Psi_{m} \otimes \Psi_{n}\right)$.

Pour tout entier $n \geqslant 1$, l'isomorphisme $\Psi_{n}$ induit une équivalence de catégories notée $\boldsymbol{\Psi}_{n}$ entre $\operatorname{Mod}\left(\mathcal{H}_{n}^{K}\right)$ et $\operatorname{Mod}\left(\mathcal{H}_{n}^{j}\right)$. On fixe un quasi-inverse $\left(\mathbf{M}_{n}^{j}\right)^{-1}$ de $\mathbf{M}_{n}^{j}$, et on note $\boldsymbol{\Phi}_{n}$ l'équivalence de catégories entre $\mathcal{R}_{n}^{K}$ et $\mathcal{R}_{n}^{\rho}$ définie par :

$$
\boldsymbol{\Phi}_{n}=\left(\mathbf{M}_{n}^{j}\right)^{-1} \circ \boldsymbol{\Psi}_{n} \circ \mathbf{M}_{n}^{K}
$$

Elle induit une bijection de $\mathcal{J}_{n}^{K}$ sur $\mathcal{J}_{n}^{\rho}$, ainsi qu'un isomorphisme de $\mathbb{Z}$-modules entre $\mathcal{G}_{n}^{K}$ et $\mathcal{G}_{n}^{\rho}$, que l'on note encore $\boldsymbol{\Phi}_{n}$. En faisant varier $n \geqslant 1$, on obtient un isomorphisme $\boldsymbol{\Phi}$ de $\mathbb{Z}$-modules gradués entre $\mathcal{G}^{K}$ et $\mathcal{G}^{\rho}$ induisant une bijection de $\mathcal{J}^{K}$ sur $\mathcal{J}^{\rho}$.

Proposition 4.5. - L'isomorphisme de $\mathbb{Z}$-modules $\boldsymbol{\Phi}$ est un isomorphisme de $\mathbb{Z}$-algèbres graduées entre $\mathcal{G}^{K}$ et $\mathcal{G}^{\rho}$. Pour tout $k \geqslant 1$, toute représentation de carré intégrable $\sigma \in \mathcal{J}^{K}$ et tout nombre réel $\alpha$ strictement compris entre 0 et $1 / 2$, on a:

$$
\begin{aligned}
\boldsymbol{\Phi}\left(T\left(1_{K^{\times}}, k\right)\right) & =T(\rho, k), \\
\boldsymbol{\Phi}(u(\sigma, k)) & =u(\boldsymbol{\Phi}(\sigma), k), \\
\boldsymbol{\Phi}(\pi(u(\sigma, k), \alpha)) & =\pi(u(\boldsymbol{\Phi}(\sigma), k), \alpha) .
\end{aligned}
$$

Démonstration. - Pour prouver que $\boldsymbol{\Phi}$ est un isomorphisme de $\mathbb{Z}$-algèbres, il suffit de montrer que, pour tous $\sigma \in \mathcal{J}_{m}^{K}$ et $\tau \in \mathcal{J}_{n}^{K}$, avec $m, n \geqslant 1$, on a $\boldsymbol{\Phi}(\sigma \times \tau)=\boldsymbol{\Phi}(\sigma) \times \boldsymbol{\Phi}(\tau)$. 
Grâce au fait 1, on peut écrire :

$$
\begin{aligned}
\mathbf{M}_{m+n}^{j}\left(\boldsymbol{\Phi}_{m+n}(\sigma \times \tau)\right) & =\boldsymbol{\Psi}_{m+n} \circ \mathbf{M}_{m+n}^{K}(\sigma \times \tau) \\
& =\boldsymbol{\Psi}_{m+n} \circ \boldsymbol{t}_{m, n}^{K}\left(\mathbf{M}_{m}^{K}(\sigma) \otimes \mathbf{M}_{n}^{K}(\tau)\right),
\end{aligned}
$$

ce qui, grâce au fait 2 , s'écrit :

$$
\boldsymbol{t}_{m, n}^{j}\left(\boldsymbol{\Psi}_{m} \mathbf{M}_{m}^{K}(\sigma) \otimes \boldsymbol{\Psi}_{n} \mathbf{M}_{n}^{K}(\tau)\right)=\boldsymbol{t}_{m, n}^{j}\left(\mathbf{M}_{m}^{j} \boldsymbol{\Phi}_{m}(\sigma) \otimes \mathbf{M}_{n}^{j} \boldsymbol{\Phi}_{n}(\tau)\right) .
$$

En employant le fait 1 derechef, on voit que le membre de droite de l'égalité ci-dessus est égal à la quantité $\mathbf{M}_{m+n}^{j}\left(\boldsymbol{\Phi}_{m}(\sigma) \times \boldsymbol{\Phi}_{n}(\tau)\right)$, ce qui prouve que $\boldsymbol{\Phi}$ est un isomorphisme de $\mathbb{Z}$-algèbres.

Pour prouver (4.9), il suffit de remarquer que le foncteur $\boldsymbol{\Phi}$ est exact et d'utiliser la propriété d'unicité de $T(\rho, k)$. Pour prouver (4.10), on pose $s=s(\Phi(\sigma))$, on note $n$ le degré de $\sigma$, c'est-à-dire l'entier tel que $\sigma \in \mathcal{J}_{n}^{K}$, et on note $\nu_{K}$ l'analogue de $\nu$ pour le groupe $\mathrm{GL}_{n}(K)$, c'est-à-dire la valeur alsolue normalisée du déterminant $\operatorname{sur} \mathrm{GL}_{n}(K)$. Il suffit alors de remarquer qu'on a :

$$
\boldsymbol{\Phi}\left(\nu_{K}^{z} \sigma\right)=\nu^{s(\sigma) z} \boldsymbol{\Phi}(\sigma)
$$

pour tout $\sigma \in \mathcal{J}^{K}$ et tout nombre complexe $z$ (voir $[\mathbf{2 8}, \S 4.2]$ ), et d'utiliser la propriété d'unicité de $u(\Phi(\sigma), k)$. La dernière égalité (4.11) en découle immédiatement.

On en déduit le résultat cherché.

Corollaire 4.6. - La conjecture U est vraie.

Démonstration. - On reprend les notations du paragraphe 4.2. D'après la proposition 4.5 et notamment (4.9), la représentation $\sigma^{\prime}=\boldsymbol{\Phi}^{-1}(\sigma)$ est de carré intégrable et isomorphe à $T\left(1_{K^{\times}}, b\right)$. D'après Tadić $[\mathbf{3 0}]$, le produit :

$$
u\left(\sigma^{\prime}, k+1\right) \times u\left(\sigma^{\prime}, k-1\right)
$$

est une représentation irréductible de $\mathrm{GL}_{2 k b}(K)$. En appliquant $\boldsymbol{\Phi}$ à (4.12), et en tenant compte de (4.10), on obtient le résultat voulu.

Remarque 4.7. - Il est possible de munir $\mathcal{G}^{\rho}$ d'une structure d'algèbre de Hopf, comme dans $[\mathbf{3 1}, \S 3]$. L'isomorphisme $\boldsymbol{\Phi}$ de la proposition 4.5 est alors un isomorphisme d'algèbres de Hopf entre $\mathcal{G}^{K}$ et $\mathcal{G}^{\rho}$. Pour voir cela, il suffit de considérer un analogue du fait 1 pour la restriction parabolique (qu'on trouve dans [14]). On montre alors, comme dans la preuve de la proposition 4.5 , que $\boldsymbol{\Phi}$ respecte la comultiplication. Elle respecte du même 
coup l'antipode qui, dans [2], est définie en fonction de l'induction et de la restriction paraboliques.

\section{Quelques conséquences de la classification}

Dans ce dernier chapitre, on présente quelques conséquences du théorème 2.6.

5.1. Décomposition de $u(\sigma, k)$ suivant la base standard. - Rappelons que l'on peut voir $\mathcal{G}=\mathcal{G}(G)$ comme le $\mathbb{Z}$-module libre engendré par l'ensemble des classes d'isomorphisme de représentations irréductibles de $G$. Ce module admet une autre base, dite standard, formée des représentations standard définies par (2.4). Il n'est pas toujours facile de connaître les coefficients de la décomposition sur la base standard de $\mathcal{G}$ d'une représentation irréductible de $G$, mais pour les représentations $u(\sigma, k)$ nous disposons de la formule suivante.

Proposition 5.1. - Soient k,l, $m \geqslant 1$ des entiers dont le produit est égal à $r$ et soit $\rho$ une représentation cuspidale unitaire de $G_{m}$. On pose $\sigma=T(\rho, l)$ et on note $W_{k}^{l}$ l'ensemble des permutations $w$ de $\{1, \ldots, k\}$ telles que $w(i)+l \geqslant i$ pour chaque $i \in\{1, \ldots, k\}$. On $a$ :

$$
u(\sigma, k)=\nu^{-\frac{k+l}{2} s(\sigma)}\left(\sum_{w \in W_{k}^{l}}(-1)^{\operatorname{sgn}(w)} \prod_{i=1}^{k} T\left(\nu^{i s(\sigma)} \rho, w(i)+l-i\right)\right)
$$

dans $\mathcal{G}$, où $\operatorname{sgn}(w) \in\{-1,1\}$ est le signe de la permutation $w$.

Ce résultat est dû à Tadić [33] lorsque $F$ est de caractéristique nulle, mais la preuve qu'il en donne (voir [33, Proposition 5.2]) montre qu'il s'agit d'une conséquence du théorème de classification 2.6 indépendamment de la caractéristique de $F$.

En théorie, la proposition 5.1 et le théorème 2.6 permettent de calculer, pour toute représentation irréductible unitaire $\pi$ de $G$, les coefficients de la décomposition de $\pi$ sur la base standard de $\mathcal{G}$.

5.2. Transfert des représentations unitaires. - Pour $n \geqslant 1$, on note $i_{n}$ l'involution de Zelevinsky-Aubert sur l'ensemble des classes de représentations irréductibles de $G_{n}$. Soient $k, n \geqslant 1$ des entiers et soit $\sigma$ une représentation de carré intégrable de $G_{n}$. On définit des entiers $a_{i}$ par (1.1) et on pose :

$$
u^{\prime}(\sigma, k)=L\left(\nu^{a_{1}} \sigma, \ldots, \nu^{a_{k}} \sigma\right),
$$


qui est une représentation irréductible de $G_{k n}$. Le théorème suivant (voir $[\mathbf{3 3}, \mathbf{6}]$ ) étend la correspondance de Jacquet-Langlands aux représentations de la forme $u(\sigma, k)$. On rappelle que $H=\mathrm{GL}_{r d}(F)$.

Théorème 5.2. - Soient $k, l, m \geqslant 1$ des entiers dont le produit est égal à $r d$, et soit $\rho$ une représentation cuspidale unitaire de $\mathrm{GL}_{m}(F)$. On pose $\sigma=T(\rho, l)$ et $\tau=T(\rho, k)$.

(1) On suppose que d divise $l m$, avec $l m=r^{\prime} d$, et on pose $\sigma^{\prime}=\mathbf{J L}(\sigma)$. Alors :

$$
\Theta_{u(\sigma, k)}(g)=(-1)^{(d-1) r^{\prime}} \Theta_{u^{\prime}\left(\sigma^{\prime}, k\right)}\left(g^{\prime}\right)
$$

pour tout couple $\left(g, g^{\prime}\right) \in H \times G$ d'éléments semisimples réguliers tels que $g \leftrightarrow g^{\prime}$.

(2) On suppose que $d$ divise $k m$, et on pose $\tau^{\prime}=\mathbf{J L}(\tau)$ et $\pi^{\prime}=i_{r}\left(u^{\prime}\left(\tau^{\prime}, l\right)\right)$. Alors :

$$
\Theta_{u(\sigma, k)}(g)=(-1)^{(k-1) l} \Theta_{\pi^{\prime}}\left(g^{\prime}\right)
$$

pour tout couple $\left(g, g^{\prime}\right) \in H \times G$ d'éléments semisimples réguliers tels que $g \leftrightarrow g^{\prime}$.

(3) On suppose que d ne divise ni $\mathrm{lm}$ ni $\mathrm{km}$. Alors :

$$
\Theta_{u(\sigma, k)}(g)=0
$$

pour tout couple $\left(g, g^{\prime}\right) \in H \times G$ d'éléments semisimples réguliers tels que $g \leftrightarrow g^{\prime}$, c'està-dire que le caractère de $u(\sigma, k)$ est nul sur les éléments semisimples réguliers de $H$ qui correspondent aux éléments semisimples réguliers de $G$.

Remarque 5.3. - Dans ce théorème, les représentations $u(\sigma, k)$ sont du côté déployé.

Comme la proposition 5.1 , ce théorème est une conséquence du théorème 2.6 indépendamment de la caractéristique de $F$. Le théorème 5.2 , joint au théorème de classification 2.6, a pour conséquence le corollaire suivant.

Corollaire 5.4. - Soit $\pi$ une représentation irréductible unitaire de H. Alors ou bien la fonction-caractère $\Theta_{\pi}$ de $\pi$ est nulle sur les éléments semisimples réguliers de $H$ qui correspondent aux éléments semisimples réguliers de $G$, ou bien il existe une représentation admissible $\pi^{\prime}$ de $G$ et un signe $\varepsilon \in\{-1,1\}$ tels que :

$$
\Theta_{\pi}(g)=\varepsilon \Theta_{\pi^{\prime}}\left(g^{\prime}\right)
$$

pour tout couple $\left(g, g^{\prime}\right) \in H \times G$ d'éléments semisimples réguliers tels que $g \leftrightarrow g^{\prime}$. La représentation $\pi^{\prime}$ est irréductible et unitaire, et sa classe d'isomorphisme est déterminée par celle de $\pi$. 
Remarque 5.5. - La proposition 5.1, le théorème 5.2 et son corollaire 5.4 sont aussi démontrés dans [6] par des méthodes différentes de celles utilisées dans [33], notamment en utilisant seulement U1 et non pas U0 qui n'était pas prouvée à ce moment-là. Cette approche fonctionne aussi en toute caractéristique, grâce au théorème 3.18.

\section{Références}

[1] J. Arthur, "The invariant trace formula, II, Global Theory", J. Amer. Math. Soc. 1 (1988), no. 3, p. 501-554.

[2] A.-M. Aubert, "Dualité dans le groupe de Grothendieck de la catégorie des représentations lisses de longueur finie d'un groupe réductif p-adique", Trans. Amer. Math. Soc. 347 (1995), no. 6, p. 2179-2189.

[3] A. I. Badulescu - "Correspondance de Jacquet-Langlands pour les corps locaux de caractéristique non nulle", Ann. Sci. École Norm. Sup. (4) 35 (2002), no. 5, p. 695-747.

[4] _ , "Un résultat d'irréductibilité en caractéristique non nulle", Tohoku Math. J. (2) 56 (2004), no. 4, p. 583-592.

[5] _ "Jacquet-Langlands et unitarisabilité", J. Inst. Math. Jussieu 6 (2007), no. 3, p. 349-379.

[6] _ _ "Global Jacquet-Langlands correspondence, multiplicity one and classification of automorphic representations", Invent. Math. 172 (2008), no. 2, p. 383-438.

[7] A. I. Badulescu \& D. A. Renard - "Sur une conjecture de Tadić", Glas. Mat. Ser. III 39(59) (2004), no. 1, p. 49-54.

[8] D. Barbasch \& A. Moy - "A unitarity criterion for p-adic groups", Invent. Math. 98 (1989), no. 1, p. 19-37.

[9] __ "Reduction to real infinitesimal character in affine Hecke algebras", J. Amer. Math. Soc. 6 (1993), no. 3, p. 611-635.

[10] J. Bernstein (rédigé par P. Deligne), "Le centre de Bernstein" in Représentations des groupes réductifs sur un corps local, coll. Travaux en cours, Hermann, Paris, 1984, p. 1-32.

$[11] \ldots$, "P-invariant distributions on $\operatorname{GL}(N)$ and the classification of unitary representations of $\operatorname{GL}(N)$ (non-Archimedean case)", Lecture Notes in Mathematics, vol. 1041, Springer, Berlin, 1984, p. 50-102.

[12] J. Bernstein, P. Deligne \& D. Kazhdan, "Trace Paley-Wiener theorem for reductive p-adic groups", J. Analyse Math. 47 (1986), p. 180-192.

[13] C. Bushnell, "Representations of reductive $p$-adic groups: localization of Hecke algebras and applications", J. London Math. Soc. (2) 63 (2001), no. 2, p. 364-386.

[14] C. J. Bushnell \& P. C. Kutzko - "Smooth representations of reductive p-adic groups: structure theory via types", Proc. London Math. Soc. (3) 77 (1998), no. 3, p. 582-634.

[15] W. CASSElman, "Introduction to the theory of admissible representations of reductive p-adic groups", disponible sur www.math.ubc.ca八〜cass/research.html. 
[16] P. Deligne, "Les corps locaux de caractéristique $p$ comme limites de corps locaux de caractéristique nulle" in Représentations des groupes réductifs sur un corps local, coll. Travaux en cours, Hermann, Paris, 1984.

[17] P. Deligne, D. Kazhdan \& M.-F. Vignéras, "Représentations des algèbres centrales simples $p$-adiques" in Représentations des groupes réductifs sur un corps local, coll. Travaux en cours, Hermann, Paris, 1984.

[18] R. Godement \& H. Jacquet, "Zeta functions of simple algebras", Lecture Notes in Mathematics, vol. 260, Springer, Berlin - New York, 1972.

[19] G. Henniart \& B. Lemaine, "Intégrales orbitales tordues sur GL $(n, F)$ et corps locaux proches : applications", Canad. J. Math. 58 (2006), no. 6, p. 1229-1267.

[20] D. Kazhdan, "Representations of groups over close local fields", J. Analyse Math. 47 (1986), p. 175-179.

[21] B. Lemaire, "Représentations génériques de $\mathrm{GL}_{N}$ et corps locaux proches", J. Algebra 236 (2001), p. 549-574.

[22] A. Mínguez, "Correspondance de Howe $\ell$-modulaire : paires duales de type II", Thèse de doctorat, Université d'Orsay, 2006.

[23] F. Rodier, "Représentations de GL $(n, k)$ où $k$ est un corps p-adique", Séminaire Bourbaki 34 (1981/82), exposé no. $\mathbf{5 8 7}$.

[24] I. Satake, "Theory of Spherical Functions on Reductive Algebraic Groups over $p$-adic Fields", Publ. Math. I.H.E.S. 18 (1963), p. 1-69.

[25] V. SÉcherre - "Représentations lisses de $\mathrm{GL}_{m}(D)$, I : caractères simples", Bull. Soc. Math. France 132 (2004), no. 3, p. 327-396.

[26] _ _Représentations lisses de $\mathrm{GL}_{m}(D)$, II : $\beta$-extensions", Compositio Math. 141 (2005), p. 1531-1550.

[27] _ "Représentations lisses de $\mathrm{GL}_{m}(D)$, III : types simples", Ann. Sci. École Norm. Sup. 38 (2005), p. 951-977.

[28] dans J. Reine Angew. Math.

[29] V. Sécherre \& S. Stevens - "Représentations lisses de $\mathrm{GL}_{m}(D)$, IV : représentations supercuspidales", J. Inst. Math. Jussieu 7 (2008), no. 3, p. 527-574.

[30] M. TADIĆ - "Classification of unitary representations in irreducible representations of general linear group (non-Archimedean case)", Ann. Sci. École Norm. Sup. (4) 19 (1986), no. 3 , p. 335-382.

[31] _ "Induced representations of $\operatorname{GL}(n, A)$ for $p$-adic division algebras $A$ ", J. Reine Angew. Math. 405 (1990), p. 48-77.

[32] _ _ "An external approach to unitary representations", Bull. Amer. Math. Soc. (N.S.) 28 (1993), no. 2, p. 215-252.

[33] _ "Representation theory of $\operatorname{GL}(n)$ over a $p$-adic division algebra and unitarity in the Jacquet-Langlands correspondence", Pacific J. Math. 223 (2006), no. 1, p. 167-200.

[34] M.-F. VignéRAs, "Induced $R$-representations of $p$-adic reductive groups", Selecta Math. (N.S.) 4 (1998), no. 4, p. 549-623. 
[35] A. Zelevinsky, "Induced representations of reductive p-adic groups II", Ann. Sci. École Norm. Sup. 13 (1980), p. 165-210.

I. Badulescu, Université de Montpellier 2, Département Mathématiques, Case Courrier 051, Place Eugène Bataillon, 34095 Montpellier Cedex, France

E-mail : badulesc@math.univ-poitiers.fr

G. Henniart, Université de Paris-Sud, Laboratoire de Mathématiques, Orsay cedex F-91405 France, CNRS, Orsay cedex F-91405 France • E-mail : Guy.Henniart@math.u-psud.fr

B. Lemaire, Institut de Mathématiques de Luminy, UMR 6206, Campus de Luminy, Case 907, 13288 Marseille Cedex 9, France • E-mail : lemaire@iml.univ-mrs.fr

V. SÉCHERRE, Université de la Méditerranée, Institut de Mathématiques de Luminy, UMR 6206, Campus de Luminy, Case 907, 13288 Marseille Cedex 9, France • E-mail : secherre@iml.univ-mrs.fr 